TRANSACTIONS OF THE

AMERICAN MATHEMATICAL SOCIETY

Volume 361, Number 2, February 2009, Pages 735-765

S 0002-9947(08)04588-1

Article electronically published on September 23, 2008

\title{
SUMS OF SQUARES AND MOMENT PROBLEMS IN EQUIVARIANT SITUATIONS
}

\author{
JAKA CIMPRIČ, SALMA KUHLMANN, AND CLAUS SCHEIDERER
}

\begin{abstract}
We begin a systematic study of positivity and moment problems in an equivariant setting. Given a reductive group $G$ over $\mathbb{R}$ acting on an affine $\mathbb{R}$-variety $V$, we consider the induced dual action on the coordinate ring $\mathbb{R}[V]$ and on the linear dual space of $\mathbb{R}[V]$. In this setting, given an invariant closed semialgebraic subset $K$ of $V(\mathbb{R})$, we study the problem of representation of invariant nonnegative polynomials on $K$ by invariant sums of squares, and the closely related problem of representation of invariant linear functionals on $\mathbb{R}[V]$ by invariant measures supported on $K$. To this end, we analyse the relation between quadratic modules of $\mathbb{R}[V]$ and associated quadratic modules of the (finitely generated) subring $\mathbb{R}[V]^{G}$ of invariant polynomials. We apply our results to investigate the finite solvability of an equivariant version of the multidimensional $K$-moment problem. Most of our results are specific to the case where the group $G(\mathbb{R})$ is compact.
\end{abstract}

\section{INTRODUCTION}

The study of positivity versus sums of squares has a long and illustrious tradition, starting with Minkowski and Hilbert. It is remarkable how the interest in such questions has risen in the last decade, in particular in fields outside real algebraic geometry. The application of semi-definite programming methods is currently turning sums of squares into an efficient tool in polynomial optimization. Another field to which sums of squares methods have recently been applied successfully is moment problems and related questions in analysis. Yet another, and still fresh, development is the study of sums of squares in non-commutative settings, and the exploration of its use in applications like engineering. An excellent overview of such ongoing developments can be found in $\mathrm{HP}$.

Both in theoretical considerations and in practical applications, it happens quite often that the situation in question allows symmetries. For example, consider the problem of characterizing all polynomials which are non-negative on a given closed subset $K$ of $\mathbb{R}^{n}$. If $K$ is invariant under some subgroup $G$ of the general linear group, one may ask for a characterization of the $G$-invariant polynomials which are non-negative on $K$. How can they be described in terms of invariant sums of squares, or even, in terms of sums of squares of invariants?

Received by the editors November 19, 2006.

2000 Mathematics Subject Classification. Primary 14P10, 14L30, 20G20.

Key words and phrases. Semi-algebraic sets, group actions, moment problems.

The third author was partially supported by the European RTNetwork RAAG, HPRN-CT2001-00271. 
The first to systematically study equivariant situations in real algebraic geometry were Procesi and Schwarz, in their landmark paper [PS]. Their main result characterizes the real orbit space of a matrix group $G$, in the case when $G$ is compact. In particular, they showed that the real orbit space is described by (finitely many) simultaneous inequalities which are essentially explicit. These results were later refined by Bröcker $[\mathrm{Br}$ ] who studied, in particular, the minimum number of inequalities needed for a description. A more recent contribution is the paper GP] by Gatermann and Parrilo, who were primarily interested in the effective aspects of sums of squares decompositions of invariant polynomials. Later we will review some of the main results of all three papers in more detail.

Apart from these works, positivity and sums of squares questions have hardly been considered in equivariant settings yet, as far as we know. One purpose of our paper is to begin a systematic study. For this reason, at least some of it is of foundational nature.

Generally, we have tried working over an arbitrary real closed base field $R$ as much as possible, instead of only over the classical real numbers $\mathbb{R}$. One reason is that the validity of a result over arbitrary $R$ often has implications for the case of base field $\mathbb{R}$, e.g. for the existence of complexity bounds, as is well known.

So our setup consists of a reductive linear group $G$ acting on an affine variety $V$, the action being defined over a real closed field $R$. Most of our results are specific to the case where the group $G(R)$ is semi-algebraically compact, that is, closed and bounded in some $G L_{N}(R)$.

After recalling and introducing a few technical notions from real algebra (Section 1), we review the main results of Procesi-Schwarz, Bröcker and GatermannParrilo in Section 2. We generalize them to arbitrary real closed base fields and give alternative, purely algebraic proofs for some parts. The main result of [GP] is generalized to the case of a compact (infinite) group of symmetries. Both our formulation and our proof are more conceptual and less matrix-based than the original account in $\mathrm{GP}$. Section 3 contains basic material. We consider an affine $G$-variety $V$ as above, with $G(R)$ semi-algebraically compact. We study the operations of contraction and extension between $R[V]$ and $R[V]^{G}$ on the cones of sums of squares and on more general quadratic modules, and also the effect of the Reynolds operator $\rho$. Also, we study the relation between quadratic modules and their associated closed sets, both in $V$ and $V / / G$. A central result proved here is that $\rho$ maps the cone $\Sigma R[V]^{2}$ of sums of squares into itself, and hence $\rho\left(\Sigma R[V]^{2}\right)=\left(\Sigma R[V]^{2}\right)^{G}$, the cone of $G$-invariant sums of squares. In fact, this characterizes the case $G(R)$ semi-algebraically compact, for in Section 4 we prove that in all other cases (with $G$ reductive) there exists $f \in R[G]$ with $\rho\left(f^{2}\right)<0$. (Here $\rho$ is the Reynolds operator of $G$ acting on itself by translation). Back to the compact case, we show by examples in Section 5 that $\left(\Sigma R[V]^{2}\right)^{G}$ usually fails to be finitely generated, as a preordering in $R[V]^{G}$. Other instructive examples are presented in this section as well.

We would like to emphasize the importance of the Reynolds operator $\rho$ (projection to the invariants), which plays a key role throughout. Its consequent use replaces the tool of invariant integration, which is available for $R=\mathbb{R}$ but not otherwise. Note that the Reynolds operator is well understood from a computational view point, not only for finite $G$ (see $[\mathrm{DK}]$ ).

The second part of the paper deals with moment problems having symmetries. Of course, the base field is now $\mathbb{R}$, the usual real numbers. For simplicity, let us 
assume that $K$ is a (basic) closed subset of $\mathbb{R}^{n}$. The $K$-moment problem asks for a characterization of all $K$-moment functionals on $\mathbb{R}[x]=\mathbb{R}\left[x_{1}, \ldots, x_{n}\right]$; that is, of all linear functionals $L: \mathbb{R}[x] \rightarrow \mathbb{R}$ arising from integration by some Borel measure on $K$ (all of whose moments exist). Suppose that $K$ is invariant under the linear group $G$. Then we may ask for a characterization of the $G$-invariant $K$-moment functionals. This task should generally be easier, compared to a characterization of all $\mathrm{K}$ moment functionals. We isolate two conditions, the invariant resp. the averaged moment property, and (IMP) resp. (AMP), under which a characterization of the $G$-invariant moment functionals is possible in a reasonable (finitistic) sense. We give various equivalent characterizations of these conditions. For example, (IMP) is equivalent to the usual moment property for the image of $K$ in the orbit space. By means of several examples we demonstrate that indeed we gain something by considering these properties: (IMP) resp. (AMP) are shown to hold in cases where the usual moment problem fails to be finitely solvable. On the other hand, we prove an equivariant version of the main result of [PSch. A large class of cases was characterized there by a geometric condition, in which the moment problem is not finitely solvable; we show that under a similar equivariant geometric condition, not even the invariant moment problem is finitely solvable.

The paper closes with a list of open questions. In a previous version of this paper we asked whether (SMP) implies (IMP) and whether (AMP) implies (IMP). Recently, both questions were answered to the negative by Tim Netzer (see, for example, Section 7.3). We would like to take this opportunity to thank him for allowing us to include his example in our paper.

\section{Notation AND PRELIMINARIES}

All rings are commutative and have a unit. For general background on real algebra and geometry we refer to $[\mathrm{BCR},[\mathrm{KS}]$ and $[\mathrm{PD}]$. In particular, background on the notion of the real spectrum may be found in these texts. We will always denote the real spectrum of a $\operatorname{ring} A$ by $\operatorname{Sper} A$.

1.1. Let $A$ be a ring. A quadratic module in $A$ is a subset $M$ of $A$ with $1 \in M$ which satisfies $M+M \subset M$ and $a^{2} M \subset M$ for every $a \in A$. A preordering in $A$ is a quadratic module $T$ which is closed under multiplication: $T T \subset T$. The smallest quadratic module in $A$ (which happens to be a preordering) is $\Sigma A^{2}$, the set of all sums of squares in $A$.

The quadratic module generated by $a_{1}, \ldots, a_{r} \in A$ in $A$ consists of all elements

$$
f=s_{0}+s_{1} a_{1}+\cdots+s_{r} a_{r}
$$

with $s_{i} \in \Sigma A^{2}$, and is denoted by $Q M_{A}\left(a_{1}, \ldots, a_{r}\right)$. The preordering generated by $a_{1}, \ldots, a_{r}$ in $A$ is the quadratic module generated by the $2^{r}$ products $a_{1}^{e_{1}} \cdots a_{r}^{e_{r}}, e_{i} \in$ $\{0,1\}$; it is denoted by $P O_{A}\left(a_{1}, \ldots, a_{r}\right)$. A quadratic module (resp., preordering) is said to be finitely generated if it can be generated by finitely many elements.

1.2. In the first part of our paper we will work over general real closed base fields $R$. By an affine $R$-variety we always mean an affine algebraic variety $V$ defined over $R$ which is reduced. Its coordinate ring is denoted $R[V]$; this is a finitely generated reduced $R$-algebra. The set of $R$-points on $V$ is denoted $V(R)$. We will use the notion of semi-algebraic subsets of $V(R)$. Recall the operator tilda (see any

of the above references): For $S$ a semi-algebraic subset of $V(R), \widetilde{S}$ is a constructible 
subset of Sper $R[V]$ and $S \mapsto \widetilde{S}$ is a bijection between semi-algebraic sets in $V(R)$ and constructible sets in Sper $R[V]$ which preserves all boolean operations. In particular, Sper $R[V]=\widetilde{V(R)}$.

1.3. Again let $A$ be a ring. Associated with a quadratic module $M$ in $A$ (or in fact with any subset $M$ of $A$ ) is the closed subset

$$
\mathcal{X}(M):=X_{A}(M):=\{\alpha \in \text { Sper } A: f(\alpha) \geq 0 \text { for every } f \in M\}
$$

of Sper $A$. If one thinks of the elements of Sper $A$ as prime cones in $A$, then $X(M)$ is the set of prime cones which contain $M$. If $M$ is finitely generated, say by $a_{1}, \ldots, a_{r}$, then $X(M)=\left\{\alpha: a_{1}(\alpha) \geq 0, \ldots, \alpha_{r}(\alpha) \geq 0\right\}$ is a constructible subset of Sper $A$.

In the geometric situation we use the following notation. Let $R$ be a real closed field and $V$ an affine $R$-variety. If $M$ is a quadratic module in $R[V]$ we write

$$
\mathcal{S}(M):=\mathcal{S}_{V}(M):=\bigcap_{f \in M}\{x \in V(R): f(x) \geq 0\}=V(R) \cap \mathcal{X}_{R[V]}(M)
$$

for the 'trace' of $\chi(M) \subset$ Sper $R[V]=\widetilde{V(R)}$ in $V(R)$. This is a closed subset of $V(R)$, which is semi-algebraic if $M$ is finitely generated.

1.4. Let $M$ be a subset of $A$. The saturation of $M$ is the preordering

$$
\operatorname{Sat}(M):=\operatorname{Sat}_{A}(M):=\{f \in A: f \geq 0 \text { on } \mathcal{X}(M)\}
$$

of $A$. We have $M \subset \operatorname{Sat}(M)$ tautologically, and $M$ is called saturated if equality holds. With every subset $X$ of Sper $A$ we can associate a saturated preordering of $A$, namely

$$
\mathcal{P}(X):=\mathcal{P}_{A}(X):=\{a \in A: a \geq 0 \text { on } X\} .
$$

The two operators $\mathcal{P}$ and $X$ set up a Galois correspondence between the subsets of Sper $A$ and the subsets of $A$, the closed objects of which are the pro-basic closed subsets of Sper $A$ on one side and the saturated preorders of $A$ on the other. In particular, we have $\operatorname{Sat}(M)=\mathcal{P}(X(M))$ for every quadratic module $M$.

See also [Sch2] for a more detailed discussion of these notions.

\section{Actions of Reductive groups on AFFine $R$-VARIEties}

2.1. Always let $R$ be a real closed field. By an $R$-variety $V$ we mean a reduced separated scheme of finite type over $R$. Most $R$-varieties in this paper will be affine. Affine $R$-varieties $V$ correspond, in a contravariant functorial way, to finitely generated $R$-algebras which are reduced (without nilpotent elements $\neq 0$ ); namely, $V$ corresponds to its coordinate ring, $R[V]$. The set of $R$-points on $V$ is denoted $V(R)$, as usual.

Always let $G$ be a linear algebraic group over $R$. The Zariski closure of $G(R)$ in $G$ is an open and closed subgroup of $G$ (of finite index), so it contains the identity component $G_{0}$ of $G$. (This is in fact true for linear groups over any ground field; see [Bo], Cor. V.18.3.) Throughout this paper we will assume that $G(R)$ is Zariski dense in $G$, or equivalently, that every connected component of $G$ contains an $R$-point.

2.2. Let $G$ act on the affine $R$-variety $V$ by means of a morphism

$$
G \times V \rightarrow V, \quad(g, x) \mapsto g x
$$

of $R$-varieties. To such an action corresponds the dual action

$$
\eta=\eta_{V}: R[V] \rightarrow R[G] \otimes_{R} R[V]
$$


which is a homomorphism of $R$-algebras. The group $G(R)$ acts on the $R$-algebra $R[V]$ through algebra automorphisms on the right by $(f, g) \mapsto f^{g}$, where $f^{g}$ is characterized by $f^{g}(x)=f(g x)$. Thus, if $\eta_{V}(f)=\sum_{i} a_{i} \otimes f_{i}$, then

$$
f^{g}=\sum_{i} a_{i}(g) \cdot f_{i}
$$

$(f \in R[V], g \in G(R))$. Using the assumption that $G(R)$ is Zariski dense in $G$, it is easy to see that an element $f \in R[V]$ is invariant under this action of $G(R)$ if and only if $\eta(f)=1 \otimes f$. One writes

$$
R[V]^{G}:=R[V]^{G(R)}=\{f \in R[V]: \eta(f)=1 \otimes f\} .
$$

This is the subring of $G$-invariants of $R[V]$.

To simplify language, we will say that a subset of $V(R)$, or of $R[V]$, is $G$-invariant if it is invariant under the action of the group $G(R)$.

2.3. Recall that a linear group $G$ over $R$ is called reductive if $G$ contains no nontrivial unipotent normal closed subgroup. (Sometimes it is also required that $G$ is connected.) Since $R$ has characteristic zero, it is equivalent that every finitedimensional $G$-module $M$ is a direct sum of irreducible $G$-modules, or equivalently, that every $G$-submodule of such $M$ has a $G$-invariant complement. (See, e.g., [DK], Theorems 2.2.13 and 2.2.5.)

Most of our results will focus on the case where $G(R)$ is semi-algebraically compact (i.e. $G(R)$ is closed and bounded in an affine $R$-space). Such a linear group $G$ over $R$ is always reductive. Indeed, $G(R)$ cannot contain any closed subgroup isomorphic to $R=\mathbb{G}_{a}(R)$ (the additive group of $R$ ), and hence $G$ cannot contain any non-trivial unipotent closed $R$-subgroup (normal or not).

When $G$ is reductive, then for any affine $G$-variety $V$ the ring $R[V]^{G}$ of $G$ invariants is finitely generated as an $R$-algebra, as was shown by Hilbert (see [DK], 2.2.10). Thus $R[V]^{G}$ is the coordinate ring of an affine $R$-variety $W$, which is called the quotient variety of $V$ by $G$ and is commonly denoted $V / / G$ :

$$
W=V / / G, \quad R[W]=R[V]^{G} .
$$

Corresponding to the inclusion $R[W]=R[V]^{G} \subset R[V]$ of $R$-algebras we have the morphism $\pi: V \rightarrow W$ of affine $R$-varieties, called the quotient morphism.

2.4. As a morphism of $R$-varieties, $\pi: V \rightarrow W=V / / G$ is surjective and open. The induced map $\pi: V(R) \rightarrow W(R)$ on $R$-points, however, fails to be surjective in general, and it is an important problem to describe its image. We will usually write

$$
Z:=\pi(V(R))
$$

for this image set. This is a semi-algebraic subset of $W(R)$.

When $G(R)$ is semi-algebraically compact, the problem of determining $Z$ has been solved by Procesi and Schwarz [PS], and complemented by Bröcker $\mathrm{Br}$. Both worked in the case $R=\mathbb{R}$, but their main results generalize to arbitrary real closed ground field:

Theorem 2.5 (Procesi-Schwarz [PS], Bröcker [Br]). Let $G$ be a linear group over $R$ acting on an affine $R$-variety $V$, and assume that $G(R)$ is semi-algebraically compact. Let $\pi: V \rightarrow V / / G=W$ be the quotient morphism.

(a) The non-empty fibres of the map $\pi: V(R) \rightarrow W(R)$ are precisely the $G(R)$-orbits in $V(R)$. 
(b) For every basic closed set $K$ in $V(R)$, the image $\pi(K)$ is a basic closed subset of $W(R)$. In particular, $Z=\pi(V(R))$ is basic closed in $W(R)$.

(c) The map $\pi: V(R) \rightarrow W(R)$ is semi-algebraically proper.

(Note that we are assuming that $G(R)$ is Zariski dense in $G$, as always.)

2.6. In the case $R=\mathbb{R}$, and for the particular case $K=V(\mathbb{R})$ in (b), this theorem is due to Procesi and Schwarz $[\mathrm{PS}]$. Bröcker $[\mathrm{Br}]$ worked over $\mathbb{R}$ as well. He extended (b) to the case of arbitrary basic closed $K$ (Proposition 5.1) and otherwise studied the question of how many inequalities are needed to describe the basic closed set $\pi(K)$.

Both $[\overline{\mathrm{PS}}$ and $\mathrm{Br}$ used transcendental arguments in their proofs (in particular, integration). Therefore the proofs do not directly generalize to other real closed ground fields. The proof of (b) from $[\mathrm{Br}]$ is easy to transfer to $R \neq \mathbb{R}$, once basic properties of the Reynolds operator have been established; we'll give it in Proposition 3.10 below. Assertion (c) follows in a standard way from (a) and (b) 11 As to assertion (a), it is possible to give an entirely algebraic argument valid over any real closed $R$. However, this uses much more structure theory of linear algebraic groups and invariant theory than the rest of this paper, and therefore we omit it. Instead we simply remark that (a) can be proved over $R \neq \mathbb{R}$ by deducing it from the case $R=\mathbb{R}$ via the Tarski principle.

2.7. A central point in the work of Procesi and Schwarz is the fact that the inequalities for the image $Z$ of $\pi: V(R) \rightarrow W(R)$ can be found constructively. Let us briefly recall how this is done. For simplicity, assume that $V$ is a linear representation of $G$, defined over $R$. Since $G(R)$ is semi-algebraically compact, there exists a $G$-invariant positive definite inner product $\langle-,-\rangle$ on $V$. Choose orthonormal linear coordinates $x_{1}, \ldots, x_{n}$ on $V$. Putting

$$
d p=\sum_{k=1}^{n} \frac{\partial p}{\partial x_{k}} d x_{k}
$$

for $p \in R[V]$, and transferring the inner product $\langle-,-\rangle$ to the cotangent bundle of $V$ by $\left\langle d x_{i}, d x_{j}\right\rangle=\delta_{i j}(i, j=1, \ldots, n)$, we have

$$
\langle d p, d q\rangle=\sum_{k=1}^{n} \frac{\partial p}{\partial x_{k}} \cdot \frac{\partial q}{\partial x_{k}}
$$

for $p, q \in R[V]$. Let $p_{1}, \ldots, p_{m}$ be generators of $R[V]^{G}$, the $R$-algebra of $G$ invariants. The inner products $\left\langle d p_{i}, d p_{j}\right\rangle(i, j=1, \ldots, m)$ are $G$-invariant, and so the symmetric matrix

$$
M=\left(\left\langle d p_{i}, d p_{j}\right\rangle\right)_{i, j=1, \ldots, m}
$$

has entries in $R[V]^{G}$. Procesi-Schwarz proved that

$$
\pi(V(R))=\{z \in W(R): M(z) \geq 0\} .
$$

(Here $M(z) \geq 0$ means that the matrix $M(z)$ is positive semi-definite.) Since $M(z) \geq 0$ if and only if all the $2^{m}-1$ principal minors of $M(z)$ are $\geq 0$, this shows that the semi-algebraic set $Z=\pi(V(R))$ is basic closed and can be described by $2^{m}-1$ non-strict inequalities.

\footnotetext{
${ }^{1}$ It is well known that a semi-algebraic map is semi-algebraically proper if it maps closed semi-algebraic sets to closed semi-algebraic sets and if its fibres are semi-algebraically compact.
} 
The actual minimal number of inequalities needed for the description of $Z$ may be much smaller. For results in this direction see $[\mathrm{Br}$.

2.8. We claim that the principal minors of the matrix $M$ above are sums of squares in $R[V]$. Indeed, $M=J J^{t}$, where $J=J a c\left(p_{1}, \ldots, p_{m}\right)=\left(\frac{\partial p_{i}}{\partial x_{k}}\right)$ is the Jacobian matrix of $\left(p_{1}, \ldots, p_{m}\right)$. From the Binet-Cauchy theorem (see Ga, for example) it follows that the principal minors of any matrix of the form $J J^{t}$ are sums of squares in the ring generated by the coefficients of $J$.

Thus, the principal minors of $M$ belong to the cone $S_{0}=\left(\Sigma R[V]^{2}\right)^{G}$ of $G$ invariant sums of squares. Below, we will study this cone more closely.

2.9. The Reynolds operator is an essential tool for working with actions of reductive groups. For later reference we collect its definition and a few basic facts.

Let $G$ be a reductive group over $R$, and let $M$ be a $G$-module which is finitedimensional or, more generally, a union of finite-dimensional submodules (such as the coordinate ring $R[V]$ of an affine $G$-variety $V$ ). Since $G$ is reductive, the module $M^{G}$ of $G$-invariants in $M$ has a $G$-invariant complement $N$, and it is immediate to see that $N$ is unique. The Reynolds operator

$$
\rho=\rho_{M}: M \rightarrow M^{G}
$$

is defined to be the projection onto the $G$-invariants along the direct sum decomposition $M=M^{G} \oplus N$.

2.10. For any affine $G$-variety $V$ we have, in particular, the Reynolds operator

$$
\rho=\rho_{V}: R[V] \rightarrow R[V]^{G}=R[V / / G] .
$$

The map $\rho$ is $R$-linear and is uniquely characterized by the following two properties:

(1) $\rho(f)=f$ for every $f \in R[V]^{G}$,

(2) $\rho\left(f^{g}\right)=\rho(f)$ for every $f \in R[V]$ and $g \in G(R)$.

(This characterization uses the fact that $G(R)$ is Zariski dense in $G$.) See [DK, 2.2.2. An important property of $\rho$ is that it is $R[V]^{G}$-linear, i.e., that

$$
\rho(a \cdot f)=a \cdot \rho(f)
$$

holds for every $a \in R[V]^{G}$ and $f \in R[V]$.

For the calculation of $\rho_{V}$ it suffices to know $\rho_{G}: R[G] \rightarrow R$, the Reynolds operator of $G$ acting on itself by translation, thanks to the following lemma:

Lemma 2.11. Let $G$ be reductive, and let $V$ be an affine $G$-variety. If $f \in R[V]$ and $\eta_{V}(f)=\sum_{i=1}^{m} a_{i} \otimes f_{i}$, then

$$
\rho_{V}(f)=\sum_{i=1}^{m} \rho_{G}\left(a_{i}\right) \cdot f_{i} .
$$

Proof. For $f \in R[V]$ with $\eta_{V}(f)=\sum_{i} a_{i} \otimes f_{i}$ define $r(f):=\sum_{i} \rho_{G}\left(a_{i}\right) \cdot f_{i}$. Then $r: R[V] \rightarrow R[V]$ is an $R$-linear map, and we'll show that $r$ satisfies properties (1) and (2) of Section 2.10. If $f \in R[V]^{G}$, then $\eta_{V}(f)=1 \otimes f$, and so $r(f)=f$. It remains to show $r\left(f^{g}\right)=r(f)$ for $f \in R[V]$ and $g \in G(R)$. By the commutative 
square

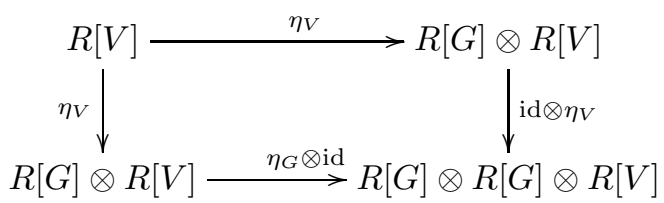

we have $\sum_{i} a_{i} \otimes \eta_{V}\left(f_{i}\right)=\sum_{i} \eta_{G}\left(a_{i}\right) \otimes f_{i}($ in $R[G] \otimes R[G] \otimes R[V]$ ). Applying the homomorphism $a \otimes b \otimes h \mapsto a(g) \cdot b \otimes h$ to both sides we get

$$
\eta_{V}\left(\sum_{i} a_{i}(g) f_{i}\right)=\sum_{i} a_{i}(g) \cdot \eta_{V}\left(f_{i}\right)=\sum_{i} a_{i}^{g} \otimes f_{i}
$$

(in $R[G] \otimes R[V]$ ). Since $f^{g}=\sum_{i} a_{i}(g) f_{i}$, we conclude

$$
r\left(f^{g}\right)=\sum_{i} \rho_{G}\left(a_{i}^{g}\right) f_{i}=\sum_{i} \rho_{G}\left(a_{i}\right) f_{i}=r(f) .
$$

If $R=\mathbb{R}$ and the group $G(\mathbb{R})$ is compact, the Reynolds operator is just averaging over the $G(\mathbb{R})$-orbits (cf. [DK], p. 45):

Proposition 2.12. Let $G(\mathbb{R})$ be compact, and let $V$ be an affine $G$-variety. Then the Reynolds operator on $V$ is characterized by

$$
\left(\rho_{V} f\right)(x)=\int_{G(\mathbb{R})} f(g x) d \lambda(g)
$$

$(f \in \mathbb{R}[V], x \in V(\mathbb{R}))$. Here $\lambda$ is the normalized Haar measure on $G(\mathbb{R})$.

Remark 2.13. In particular, if the group $G$ is finite, then

$$
\rho_{V}(f)=\frac{1}{|G|} \sum_{g \in G(R)} f^{g}
$$

for every $f \in R[V]$. Of course, this is true for any $R$ and is not restricted to $R=\mathbb{R}$.

Lemma 2.14. For every equivariant morphism $\varphi: V \rightarrow V^{\prime}$ of affine $G$-varieties, the square

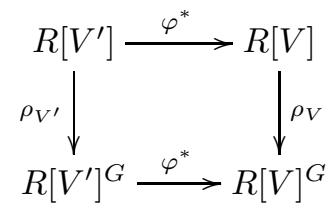

commutes.

Proof. There are unique $G$-submodules $M$ of $R[V]$ and $M^{\prime}$ of $R\left[V^{\prime}\right]$ such that $R[V]=R[V]^{G} \oplus M$ and $R\left[V^{\prime}\right]=R\left[V^{\prime}\right]^{G} \oplus M^{\prime}$. The map $\varphi^{*}: R\left[V^{\prime}\right] \rightarrow R[V]$ is a $G$-module homomorphism and hence respects these decompositions. The claim follows from this.

2.15. Let the reductive group $G$ act on the affine $R$-variety $V$, and let $\omega$ be the isomorphism type of a finite-dimensional irreducible $G$-module. A polynomial $f \in$ $R[V]$ is said to be semi-invariant of type $\omega$ if the $G$-submodule of $R[V]$ generated by $f$ is isomorphic to a direct sum of copies of $\omega$. 
The following generalizes a theorem by Gatermann and Parrilo ([GP, Theorems 5.3 and 6.2 ):

Theorem 2.16. Let $V$ be an affine $G$-variety, and assume that $G(R)$ is semialgebraically compact. Let $f \in R[V]$ be a $G$-invariant sum of squares. Then $f$ can be written

$$
f=f_{1}^{2}+\cdots+f_{m}^{2}
$$

in such a way that every $f_{i} \in R[V]$ is semi-invariant (of some type $\omega_{i}$ ).

In short: An invariant sum of squares is a sum of squares of semi-invariants. (Note that conversely it is not true that every sum of squares of semi-invariants is invariant.)

The main result of [GP] corresponds to the case of Theorem 2.16 where $G$ is finite and $V$ is a linear representation of $G$.

2.17. The proof follows the ideas of GP. However, we have translated them into a more conceptual and less matrix-based setting. It uses what has been called the "Gram matrix method" [PW], for the characterization of sums of squares of polynomials. We start by reviewing and rephrasing this approach.

At the beginning, $k$ can be any field of characteristic not two. Let $V$ be a finite-dimensional $k$-vector space, and let

$$
S(V)=\bigoplus_{n \geq 0} S^{n}(V)
$$

be the symmetric algebra of $V$, where $S^{n}(V)$ is the $n$-th symmetric power of $V$. Let $V^{\vee}$ be the dual vector space of $V$. The symmetric algebra

$$
S\left(V^{\vee}\right)=\bigoplus_{n \geq 0} S^{n}\left(V^{\vee}\right)
$$

of $V^{\vee}$ is canonically identified with the ring of polynomials on $V$; namely, $S^{n}\left(V^{\vee}\right)$ is identified with the homogeneous polynomials of degree $n$.

Fix a degree $d \geq 0$. Any (symmetric) bilinear form

$$
\gamma: S^{d}(V) \times S^{d}(V) \rightarrow k
$$

determines a homogeneous polynomial $p_{\gamma}$ on $V$ of degree $2 d$, that is, an element of $S^{2 d}\left(V^{\vee}\right)$. Indeed, $\gamma$ is an element of

$$
\left(S^{d}(V) \otimes S^{d}(V)\right)^{\vee}=S^{d}(V)^{\vee} \otimes S^{d}(V)^{\vee}=S^{d}\left(V^{\vee}\right) \otimes S^{d}\left(V^{\vee}\right),
$$

and applying the product map $S^{d}\left(V^{\vee}\right) \otimes S^{d}\left(V^{\vee}\right) \rightarrow S^{2 d}\left(V^{\vee}\right)$ to this element yields $p_{\gamma}$. As a map from $V$ to $k, p_{\gamma}$ is given by

$$
p_{\gamma}(v)=\gamma\left(v^{d}, v^{d}\right) \quad(v \in V),
$$

where $v^{d}$ denotes the $d$-th power in the symmetric algebra $S(V)$. In analogy to the terminology in [PW], we'll say that $\gamma$ is a Gram form for the homogeneous polynomial $f$ (of degree $2 d$ ) if $p_{\gamma}=f$.

Now assume that $k=R$ is a real closed field. Then a homogeneous polynomial $f \in S^{2 d}\left(V^{\vee}\right)$ (of degree $2 d$ ) is a sum of squares of (homogeneous) polynomials (of degree $d$ ) if and only if $f$ has a Gram form which is positive semi-definite (psd). In fact, $f$ is a sum of $r$ squares if and only if $f$ has a psd Gram form of rank $\leq r$. Note that the set of Gram forms of $f$ is an affine-linear subspace of $S^{2}\left(S^{d}\left(V^{\vee}\right)\right)$, 
the space of symmetric bilinear forms on $S^{d}(V)$. The set of psd Gram forms of $f$ is therefore a closed convex semi-algebraic subset of $S^{2}\left(S^{d}\left(V^{\vee}\right)\right)$.

2.18. We now give the proof of Theorem 2.16 Since $V$ has an equivariant closed embedding into a linear representation $W$ of $G$, and since $R[W]^{G} \rightarrow R[V]^{G}$ is surjective, it is clear that we can assume that $V$ is a linear representation of $G$, i.e. a finite-dimensional $R$-vector space with a linear $G$-action. We will give the proof for forms, i.e. homogeneous polynomials. This is not a restriction of generality.

Thus assume $f$ is a homogeneous polynomial on $V$ of degree $2 d$ which is $G$ invariant and which is a sum of squares of polynomials. Thus $f$ has a psd Gram form $\gamma \in S^{2}\left(S^{d}\left(V^{\vee}\right)\right)$, which is a psd symmetric bilinear form

$$
\gamma: S^{d}(V) \times S^{d}(V) \rightarrow R
$$

with $p_{\gamma}=f$ (see Section 2.17). Consider the action of $G$ on $S^{2}\left(S^{d}\left(V^{\vee}\right)\right)$ which is induced by its action on $V$. For every $g \in G(R), \gamma^{g}$ is again a psd Gram form of $f$. Since the set of psd Gram forms of $f$ is convex, it contains a $G$-invariant element by Proposition 3.5 below 2 For what follows, we can therefore assume that $\gamma$ is $G$-invariant.

Consider the decomposition of the $G$-module $S^{d}(V)$ into isotypical components:

$$
S^{d}(V)=M_{1} \oplus \cdots \oplus M_{r} .
$$

Thus the $M_{i}$ are $G$-invariant, and $\operatorname{Hom}_{G}\left(M_{i}, M_{j}\right)=0$ for $i \neq j$. The group $G(R)$ being semi-algebraically compact, every irreducible representation of $G$ is self-dual, i.e., isomorphic to its dual. (Choose a $G$-invariant positive inner product to see this.) Hence $M_{i}^{\vee} \cong M_{i}$ as $G$-modules, for each $i$.

The decomposition (11) is orthogonal with respect to $\gamma$; that is, $\gamma(x, y)=0$ for all $x \in M_{i}$ and $y \in M_{j}$ whenever $i \neq j$. Indeed, by the $G$-invariance of $\gamma$ we have $\gamma(x, y)=\gamma(g x, g y)$ for all $x, y \in S^{d}(V)$ and $g \in G(R)$. In particular, the linear map $M_{j} \rightarrow M_{i}^{\vee}$ induced by the restriction of $\gamma$ to $M_{i} \oplus M_{j}$ is $G$-equivariant. Since $M_{i}^{\vee} \cong M_{i}$ as $G$-modules, as mentioned before, it follows that $M_{j} \rightarrow M_{i}^{\vee}$ must be the zero map, which means that $M_{i}$ and $M_{j}$ are orthogonal.

Diagonalizing the restriction of $\gamma$ to each $M_{i}$ separately, we see from this that $f$ can be written as a sum of squares of semi-invariant polynomials.

\section{QuAdratic Modules AND SEMI-AlgEbraiC SETS IN THE ORBIT VARIETy}

3.1. When studying quadratic modules $M$ in the coordinate ring of an affine $R$ variety $V$, it is usually necessary to work with the (pro-basic and closed) subsets $X(M)$ in the real spectrum of $R[V]$, rather than with their traces $\mathcal{S}(M)$ in $V(R)$ (see Section 1.3 for the notation), unless one knows that the quadratic module $M$ is finitely generated. Since we cannot always assume this, we are using the real spectrum.

To have a notation available which is less awkward, let us introduce the following shorthands. Let $V$ be an affine $R$-variety with coordinate ring $R[V]$, and let $M$ be a quadratic module in $R[V]$. We write

$$
\chi_{V}(M):=X_{R[V]}(M)
$$

\footnotetext{
${ }^{2}$ This forward reference is for ease of exposition only and does not create a logical circle.
} 
for the closed subset of $\widetilde{V(R)}=$ Sper $R[V]$ which is associated with $M$. Given a subset $X$ of $\widetilde{V(R)}$, we write

$$
\mathcal{P}_{V}(X):=\mathcal{P}_{R[V]}(X)
$$

for the cone of elements in $R[V]$ that are non-negative on $X$.

3.2. In the following, always let $G$ be a reductive group over $R$, let $V$ be an affine $G$-variety and $\pi: V \rightarrow V / / G=W$ the quotient morphism. By $\pi$ we also denote the induced map $\pi: V(R) \rightarrow W(R)$ on real points. We will always write $Z:=\pi(V(R))$ for the image set of $\pi: V(R) \rightarrow W(R)$. This is a semi-algebraic subset of $W(R)$. By a theorem of Luna $[\mathrm{Lu}, Z$ is closed in $W(R)$. (We will not use this fact.) Accordingly,

$$
\tilde{\pi}: \operatorname{Sper} R[V]=\widetilde{V(R)} \rightarrow \widetilde{W(R)}=\operatorname{Sper} R[W]
$$

denotes the associated map of real spectra. The image of $\widetilde{\pi}$ is $\widetilde{Z}$.

The following observations are pure formalities and have nothing to do with the specific situation:

Lemma 3.3. Let $M$ be an arbitrary quadratic module in $R[V]$. Put $N:=M \cap R[W]$, and write $X:=\mathcal{X}_{V}(M)$ and $Y:=\mathcal{X}_{W}(N)$.

(a) $\tilde{\pi}(X) \subset Y$.

(b) If $M$ is saturated in $R[V]$, then $N$ is saturated in $R[W]$. In fact, $N=$ $\mathcal{P}_{W}(\tilde{\pi}(X))$, and hence $\widetilde{\pi}(X)=Y$.

(c) Equality $X=\widetilde{\pi}^{-1}(Y)$ holds if and only if $M \subset \operatorname{Sat}_{V}(N)$. In particular, then, $\widetilde{\pi}(X)=\widetilde{Z} \cap Y$.

If $M$ is saturated in $R[V]$, then (b) implies that $Y$ is the pro-basic closed hull of $\widetilde{\pi}(X)$. One instance when the equivalent conditions of (c) are satisfied is when $M$ is generated by elements of $R[W]$, as a quadratic module in $R[V]$.

Proof. Denote the inclusion $R[W] \subset R[V]$ by $i$. (a) is obvious. To prove (b), note that (a) says $N \subset \mathcal{P}_{W}(\widetilde{\pi}(X))$. Conversely let $b \in \mathcal{P}_{W}(\widetilde{\pi}(X))$. Then $i(b) \geq 0$ on $X$, so $i(b) \in \mathcal{P}_{V}(X)=M$, which means $b \in N$. To prove (c), note that $X_{V}(N)=\widetilde{\pi}^{-1}(Y)$. From $N \subset M$ it follows that $\operatorname{Sat}_{V}(N) \subset \operatorname{Sat}_{V}(M)$. Hence $M \subset \operatorname{Sat}_{V}(N) \Leftrightarrow \operatorname{Sat}_{V}(M)=\operatorname{Sat}_{V}(N) \Leftrightarrow X=\widetilde{\pi}^{-1}(Y)$.

For more interesting results, we have to assume that $G(R)$ is semi-algebraically compact.

Theorem 3.4. Assume that $G(R)$ is semi-algebraically compact. Then for every $G$-invariant convex subset $C$ of $R[V]$, the Reynolds operator $\rho_{V}$ satisfies $\rho_{V}(C)=$ $C \cap R[V]^{G}$. In particular, if $C$ is non-empty, then $C$ contains a $G$-invariant element.

Since $\rho_{V}$ is the identity on $R[V]^{G}$, the inclusion $C \cap R[V]^{G} \subset \rho_{V}(C)$ is trivial, and we only have to show $\rho_{V}(C) \subset C$. The proof is obvious when $G$ is finite, since then $\rho_{V}(f)$ is a convex combination of the finitely many $G$-translates of $f$ (Remark 2.13). In the general case, invariant integration may replace this argument when $R=\mathbb{R}$, but not for other $R$.

Instead we use the following uniform argument. Let $f \in C$. There is a finitedimensional $G$-invariant subspace $U$ of $R[V]$ containing $f$. So the theorem follows from the following. 
Proposition 3.5. Assume that $G(R)$ is semi-algebraically compact, and let $U$ be a finite-dimensional G-module. Let $\rho_{U}: U \rightarrow U^{G}$ be the Reynolds operator of the $G$-module $U$. Then $\rho_{U}(u)$ lies in the convex hull of the orbit $G(R) u$, for every $u \in U$.

In particular, if $C$ is a $G$-invariant convex subset of $U$, then $\rho_{U}(C)=C \cap U^{G}$.

Proof. There exists a $G$-invariant positive definite inner product on $U$. By Carathéodory's lemma, the convex hull $C$ of the orbit $G(R) u$ is semi-algebraically compact. Hence there exists a unique point $v$ in $C$ of minimal distance to the origin. Clearly, $v$ must be $G$-invariant. Being a convex combination of finitely many translates $g u, g \in G(R)$, it is clear that in fact $v=\rho(u)$.

Corollary 3.6. Let $G(R)$ be semi-algebraically compact, let $V$ be an affine $G$ variety, and let $W=V / / G$. Also, let $\rho=\rho_{V}: R[V] \rightarrow R[W]$.

(a) $\rho\left(\Sigma R[V]^{2}\right)=R[W] \cap \Sigma R[V]^{2}=\left(\Sigma R[V]^{2}\right)^{G}$. This is a preordering in $R[W]$, and we denote it by $S_{0}$.

(b) If $M$ is any quadratic module in $R[V]$, then $\rho(M)$ is an $S_{0}$-module in $R[W]$.

(c) If $M$ is a G-invariant quadratic module in $R[V]$, then $\rho(M)=M \cap R[W]$.

Proof. For any $G$-invariant quadratic module $M$ in $R[V]$ we have $\rho(M)=M \cap R[W]$ by Theorem 3.4. This proves (a) and (c). To prove (b), let $M$ be an arbitrary quadratic module in $R[V]$. Clearly $\rho(M)$ is additively closed. For every $f \in S_{0}$ we have $f M \subset M$ since $f \in \Sigma R[V]^{2}$. Hence $f \cdot \rho(M)=\rho(f M) \subset \rho(M)$.

Corollary 3.7. Let $G(R)$ be semi-algebraically compact, and let $V$ be an affine $G$ variety. The elements of $S_{0}=\left(\Sigma R[V]^{2}\right)^{G}$ are precisely the finite sums of elements of the form $\rho\left(a^{2}\right)$ with $a \in R[V]$ semi-invariant (cf. Section 2.15).

Proof. Let $f \in S_{0}$, so $f$ is a $G$-invariant sum of squares in $R[V]$. By Theorem 2.16 we can write $f=f_{1}^{2}+\cdots+f_{r}^{2}$ with each $f_{i}$ semi-invariant. Therefore

$$
f=\rho(f)=\rho\left(f_{1}^{2}\right)+\cdots+\rho\left(f_{r}^{2}\right) .
$$

Remark 3.8. In Section 5, we will give examples showing the following:

(1) The preordering $S_{0}$ in $R[W]$ need not be finitely generated (Example 5.3 .

(2) If $T$ is a finitely generated preordering in $R[V]$, then the $S_{0}$-module $\rho(T)$ need not be finitely generated, not even if $T$ is $G$-invariant (Example $5.5)$.

(3) If $T$ is a preordering in $R[V]$, then the quadratic module $\rho(T)$ in $R[W]$ need not be a preordering (Example 5.7).

All these examples have the simplest possible group acting, namely the group $G$ of order two.

Corollary 3.9. Let $G(R)$ be semi-algebraically compact, and let $K$ be a $G$-invariant subset of $V(R)$. If $f \in R[V]$ satisfies $f(x) \geq 0$ for every $x \in K$, then $(\rho f)(\pi x) \geq 0$ for every $x \in K$. In other words,

$$
\mathcal{P}_{V}(K)^{G}=\rho\left(\mathcal{P}_{V}(K)\right)=\mathcal{P}_{W}(\pi K) .
$$


Proof. $\mathcal{P}_{V}(K)$ is a $G$-invariant convex subset of $R[V]$, and $\mathcal{P}_{V}(K) \cap R[W]=$ $\mathcal{P}_{W}(\pi K)$. Therefore, the corollary is a special case of Theorem 3.4. Alternatively, it follows from Lemma 3.3(b) applied to $M=\mathcal{P}_{V}(K)$, using Corollary 3.6(c).

We now recall Bröcker's result that $\pi: V(R) \rightarrow W(R)$ maps $G$-invariant basic closed sets to basic closed sets. The proof in $[\mathrm{Br}]$ uses integration, and so it works only for $R=\mathbb{R}$. Using Corollary 3.9, however, there is no difficulty in giving a proof which works in general:

Proposition 3.10. Assume that $G(R)$ is semi-algebraically compact. Let $f_{1}, \ldots, f_{r}$ $\in R[V]$, and let $K:=\mathcal{S}_{V}\left(f_{1}, \ldots, f_{r}\right)$ and $T:=P O_{V}\left(f_{1}, \ldots, f_{r}\right)$. If $K$ is $G$ invariant, then the semi-algebraic set $\pi(K)$ in $W(R)$ is basic closed. In fact,

$$
\widetilde{\pi(K)}=X_{W}(\rho(T))=\bigcap_{a \in R[V]} \bigcap_{i \in\{0,1\}^{r}} X_{W}\left(\rho\left(a^{2} f_{1}^{i_{1}} \cdots f_{r}^{i_{r}}\right)\right),
$$

and equality holds for a finite sub-intersection of the right hand intersection.

Proof (Cf. [Br], Prop. 1.2, Prop. 5.1). $T \subset \mathcal{P}_{V}(K)$ implies $\rho(T) \subset \rho\left(\mathcal{P}_{V}(K)\right)=$ $\mathcal{P}_{W}(\pi K)$ (Corollary [3.9), and thus $\pi(K) \subset X_{W}(\rho(T))$. Conversely let $\beta \in \widehat{W(R)}$, $\beta \notin \widetilde{\pi(K)}$. Thus $\widetilde{\pi}^{-1}(\beta) \cap \mathcal{X}_{V}(T)=\varnothing$. By an application of the general Stellensatz ([BCR], p. 91, [KS], p. 143), this means that the preordering of $R[V]$ generated by $P_{\beta}:=\{b \in R[W]: b(\beta) \geq 0\}$ and by $T$ contains an element $-b$ with $b \in R[W]$, $b(\beta)>0$. So there is an identity

$$
-b=\sum_{j=1}^{m} b_{j} t_{j}
$$

in $R[V]$, where $b \in R[W]$ satisfies $b(\beta)>0$, and where $b_{j} \in P_{\beta}$ and $t_{j} \in T$. Applying $\rho$ gives $-b=\sum_{j} b_{j} \rho\left(t_{j}\right)$, which shows that $\rho\left(t_{j}\right)(\beta)<0$ for some $j$. Since $t_{j}$ is a sum of finitely many products $a^{2} f_{1}^{i_{1}} \cdots f_{r}^{i_{r}}$ with $i_{\nu} \in\{0,1\}$ and $a \in R[V]$, we see that $\beta$ is not contained in the right hand intersection.

A simple compactness argument implies that $\widetilde{\pi(K)}$ is equal to a finite subintersection of the double intersection. Namely, the sets in the double intersection are clearly constructible, while $\widetilde{\pi(K)}$ is constructible because $\pi(K)$ is semi-algebraic by the Tarski-Seidenberg Theorem. The claim now follows from the fact that the constructible sets are closed and compact in the constructible topology.

Corollary 3.11. Assume that $G(R)$ is semi-algebraically compact, and let $S_{0}=$ $\rho\left(\Sigma R[V]^{2}\right)$ as above. Then $\mathcal{X}_{W}\left(S_{0}\right)=\widetilde{Z}$.

Proof. This is the particular case $T=\Sigma R[V]^{2}$ of Proposition 3.10 see Corollary 3.6. A stronger (explicit) result can be obtained from Sections 2.7 and 2.8 .

The next result generalizes this corollary (Corollary 3.11 corresponds to the case $N=\Sigma R[W]^{2}$ and $\left.M=\Sigma R[V]^{2}\right)$ :

Proposition 3.12. Let $G(R)$ be semi-algebraically compact. Let $N$ be a quadratic module in $R[W]$, and let $M$ be the quadratic module which is generated by $N$ in $R[V]$.

(a) $\rho(M)=M \cap R[W]$, and this is the $S_{0}$-module generated by $N$ in $R[W]$.

(b) $\widetilde{\pi}\left(X_{V}(M)\right)=X_{W}(N) \cap \widetilde{Z}=X_{W}(M \cap R[W])$. 
(c) In particular, if $N$ is finitely generated, then $X_{W}(M \cap R[W])$ is constructible in $\widehat{W(R)}$.

Proof. (a) Clearly, $M$ is $G$-invariant. Hence $\rho(M)=M \cap R[W]$, and this is an $S_{0}$-module (Corollary 3.6). Every element $f \in M$ can be written $f=\sum_{i} a_{i}^{2} g_{i}$ with $g_{i} \in N$ and $a_{i} \in R[V]$. Hence

$$
\rho(f)=\sum_{i} \rho\left(a_{i}^{2}\right) g_{i}
$$

which shows that $\rho(M)$ is contained in the $S_{0}$-module generated by $N$.

For the proof of (b) note that $X_{V}(M)=\widetilde{\pi}^{-1}\left(\mathcal{X}_{W}(N)\right)$, from which we get the first equality in (b). By (a), $\chi_{W}(M \cap R[W])=\mathcal{X}_{W}(N) \cap \mathcal{X}_{W}\left(S_{0}\right)$, and combined with Corollary 3.11 this gives the second equality. (c) is obvious from (b).

Corollary 3.13. A quadratic module $N$ in $R[W]$ is of the form $N=M \cap R[W]$ for some quadratic module $M$ in $R[V]$, if and only if $N$ is an $S_{0}$-module.

Proof. If $N=M^{\prime} \cap R[W]$ with some quadratic module $M^{\prime}$ in $R[V]$, then also $N=M \cap R[W]$ with $M$ the quadratic module generated by $N$ in $R[V]$, and so $N$ is an $S_{0}$-module by Proposition 3.12(a). Conversely, if $N$ is an $S_{0}$-module, let $M$ be the quadratic module generated by $N$ in $R[V]$; then $N=M \cap R[W]$, again by Proposition 3.12 (a).

Remark 3.14. Proposition 3.10 implies in particular that every $G$-invariant basic closed set $K \subset V(R)$ has a description $K=\mathcal{S}_{V}\left(h_{1}, \ldots, h_{m}\right)$ by $G$-invariant functions $h_{1}, \ldots, h_{m} \in R[V]$. More precisely, if $K=\mathcal{S}_{V}\left(f_{1}, \ldots, f_{r}\right)$ with arbitrary $f_{i} \in R[V]$, then the $h_{j}$ can be chosen of the form $\rho\left(a^{2} f_{1}^{i_{1}} \cdots f_{r}^{i_{r}}\right)$ with $a \in R[V]$ and $i_{\nu} \in\{0,1\}$.

On the other hand, it is not true in general that $K=\mathcal{S}_{V}\left(\rho f_{1}, \ldots, \rho f_{r}\right)$. (Only " $\subset$ " holds in general, by Corollary [3.9). For example, consider $G=\mu_{2}$ acting on the line $V=\mathbb{A}^{1}$ through multiplication by -1 , and take $K=[-1,1]=\mathcal{S}_{V}\left(f_{1}, f_{2}\right)$, where $f_{1}=1+x, f_{2}=1-x$. Then $\rho f_{i}=1(i=1,2)$, so $\mathcal{S}_{V}\left(\rho f_{1}, \rho f_{2}\right)=R \neq K$.

Therefore the question arises as to how $G$-invariant functions $h_{1}, \ldots, h_{r}$ with $K=\mathcal{S}_{V}\left(h_{1}, \ldots, h_{r}\right)$ can be found concretely. Here we give an answer in the case where $G$ is finite:

Proposition 3.15. Assume $|G|=n$ is finite, and let $f_{1}, \ldots, f_{r} \in R[V]$ be such that the set $K=\mathcal{S}_{V}\left(f_{1}, \ldots, f_{r}\right)$ is $G$-invariant. For $i=1, \ldots, r$ and $j=1, \ldots, n$ let $s_{i j}$ be the $j$-th elementary symmetric function in the $n$ elements $f_{i}^{g}(g \in G)$. Then $s_{i j} \in R[V]^{G}$ and $K$ is the subset of $V(R)$, where the $n r$ functions $s_{i j}$ are non-negative.

Proof. Given real numbers $x_{1}, \ldots, x_{n}$, let $s_{i}=s_{i}\left(x_{1}, \ldots, x_{n}\right)$ be the $i$-th elementary symmetric function of the $x_{j}$. Then

$$
x_{1}, \ldots, x_{n} \geq 0 \quad \Leftrightarrow \quad s_{1}, \ldots, s_{n} \geq 0 .
$$

Indeed, the right hand condition implies $\prod_{j}\left(x-x_{j}\right)=x^{n}-s_{1} x^{n-1}+\cdots+(-1)^{n} s_{n} \neq$ 0 for any $x<0$, since all summands have the same sign. Hence for every index $i=1, \ldots, r$,

$$
\bigcap_{g \in G}\left\{f_{i}^{g} \geq 0\right\}=\bigcap_{j=1}^{n}\left\{s_{i j} \geq 0\right\}
$$

and so $K=\bigcap_{i} \bigcap_{g \in G}\left\{f_{i}^{g} \geq 0\right\}=\bigcap_{i} \bigcap_{j}\left\{s_{i j} \geq 0\right\}$. 
Remark 3.16. For any basic closed and $G$-invariant subset $K$ of $V(R)$, Proposition 3.15, combined with explicit inequalities for $\pi(V(R)$ ) (Section 2.7), gives a constructive way for obtaining inequalities describing $\pi(K)$ in the orbit variety.

We do not know whether a similar constructive procedure exists for the case $G(R)$ semi-algebraically compact, but infinite.

\section{Reynolds operator and sums of Squares in the NON-COMPACT CASE}

Let $G$ be a reductive group over $R$ and $V$ an affine $G$-variety with Reynolds operator $\rho: R[V] \rightarrow R[V]^{G}$. If $G(R)$ is semi-algebraically compact, we have seen that $\rho\left(f^{2}\right)$ is a sum of squares in $R[V]$, for every $f \in R[V]$, and in turn, that

$$
\rho\left(\Sigma R[V]^{2}\right)=\left(\Sigma R[V]^{2}\right)^{G}
$$

(see Corollary 3.6). In this section we will prove that this key property fails (for suitable $V$ ) whenever $G(R)$ is not semi-algebraically compact. First, we need two lemmas:

Lemma 4.1. Let $G$ be a reductive group over $R$, and let $\rho_{G}: R[G] \rightarrow R$ be the Reynolds operator. The following conditions are equivalent:

(i) $\rho_{G}\left(f^{2}\right) \geq 0$ for every $f \in R[G]$;

(ii) for every affine $G$-variety $V$ one has $\rho_{V}\left(\Sigma R[V]^{2}\right) \subset \Sigma R[V]^{2}$.

For brevity, we will say that $G$ has property ( $\star$ ) if (i) and (ii) hold.

Proof. Of course, (i) is a particular case of (ii). Assume that (i) holds, and let $f \in R[V]$, with (say) $\eta_{V}(f)=\sum_{i=1}^{m} a_{i} \otimes f_{i}$ in $R[G] \otimes R[V]$. Hence

$$
\eta_{V}\left(f^{2}\right)=\sum_{i, j=1}^{m}\left(a_{i} a_{j}\right) \otimes\left(f_{i} f_{j}\right)
$$

and therefore

$$
\rho<_{V}\left(f^{2}\right)=\sum_{i, j=1}^{m} \rho_{G}\left(a_{i} a_{j}\right) \cdot f_{i} f_{j}
$$

(Lemma 2.11). The symmetric matrix $S:=\left(\rho_{G}\left(a_{i} a_{j}\right)\right)_{i, j=1, \ldots, m}$ over $R$ is positive semi-definite, since for $c_{1}, \ldots, c_{m} \in R$ we have

$$
\sum_{i, j=1}^{m} c_{i} c_{j} \rho_{G}\left(a_{i} a_{j}\right)=\rho_{G}\left(\sum_{i, j=1}^{m} c_{i} c_{j} a_{i} a_{j}\right)=\rho_{G}\left(\left(\sum_{i=1}^{m} c_{i} a_{i}\right)^{2}\right) \geq 0
$$

by hypothesis (i). After diagonalizing the matrix $S$, one therefore sees that $\rho_{V}\left(f^{2}\right)$ is a sum of squares in $R[V]$.

Remark 4.2. In Corollary 3.6 it was proved that property ( $\star$ ) holds whenever $G(R)$ is semi-algebraically compact. For $R=\mathbb{R}$, the usual real numbers, there is an even easier transcendental proof, which uses characterization (i) from Lemma 4.1 Indeed,

$$
\rho_{G}\left(f^{2}\right)=\int_{G(\mathbb{R})} f(g)^{2} d g \geq 0
$$

(see Proposition 2.12) is immediate for $f \in \mathbb{R}[G]$. 
Example 4.3. The multiplicative group $G=\mathbb{G}_{m}$ does not have property ( $($ ). Indeed, let $R\left[\mathbb{G}_{m}\right]=R\left[x, x^{-1}\right]$ be its coordinate ring. The Reynolds operator $\rho: R\left[\mathbb{G}_{m}\right] \rightarrow R$ is given by

$$
\rho: \sum_{i \in \mathbb{Z}} a_{i} x^{i} \mapsto a_{0}
$$

$\left(a_{i} \in R\right)$. In particular, $\rho\left(\left(x-x^{-1}\right)^{2}\right)=-2$, and so property $(\star)$ fails.

Lemma 4.4. Let $G$ be reductive, let $V$ be a homogeneous affine $G$-variety, and consider the Reynolds operator $\rho=\rho_{V}: R[V] \rightarrow R$. If $\rho\left(f^{2}\right) \geq 0$ for every $f \in$ $R[V]$, then actually $\rho\left(f^{2}\right)>0$ for every $f \neq 0$.

Recall that a $G$-variety $V \neq \varnothing$ is called homogeneous if the group $G(R(\sqrt{-1}))$ acts transitively on the set $V(R(\sqrt{-1}))$.

Proof. We first prove that the set

$$
I:=\left\{f \in R[V]: \rho\left(f^{2}\right)=0\right\}
$$

is an ideal in $R[V]$. Indeed, for $f \in I$ and $b \in R[V]$ we have

$$
0 \leq \rho\left((b+t f)^{2}\right)=\rho\left(b^{2}\right)+2 t \cdot \rho(b f)
$$

for every $t \in R$, which implies $\rho(b f)=0$. Hence $a I \subset I$ for every $a \in R[V]$ (take $\left.b:=a^{2} f\right)$. Moreover, if $f, g \in I$, then $\rho\left((f \pm g)^{2}\right)= \pm 2 \rho(f g) \geq 0$, hence $\rho\left((f \pm g)^{2}\right)=0$, and so we have shown that $I$ is an ideal.

If $f \in I$ and $g \in G(R)$, then also $f^{g} \in I$, since $\rho\left(\left(f^{g}\right)^{2}\right)=\rho\left(\left(f^{2}\right)^{g}\right)=\rho\left(f^{2}\right)=0$ by the $G$-invariance of $\rho$. Therefore the closed subvariety of $V$ defined by $\sqrt{I}$ is invariant under translation by $G(R)$, and hence must be equal to $V$ since $1 \notin I$. Thus $I=(0)$.

Now we can show:

Theorem 4.5. Let $G$ be a reductive group over $R$. If $G$ has property $(\star)$, then $G(R)$ is semi-algebraically compact.

The converse has already been proved in Corollary 3.6.

Proof. We assume that $G(R)$ is non-compact and shall arrive at a contradiction. Since $G(R)$ is not compact, $G$ contains a split torus, i.e. a closed $R$-subgroup $H$ isomorphic to $\mathbb{G}_{m}$. Consider the natural left action $(h, g) \mapsto h g$ of $H$ on $G$ by translation, and let $H \backslash G$ be the quotient variety. It is known ([KH], Thm. 5.1) that $H \backslash G$ is an affine variety, with $R[H \backslash G]=R[G]^{H}$ the ring of $H$-invariants in $R[G]$ with respect to this action. Now $H \backslash G$ is a homogeneous $G$-variety for the (left) action

$$
G \times(H \backslash G) \rightarrow H \backslash G, \quad(g, H x) \mapsto H x g^{-1},
$$

and the Reynolds operator factors as

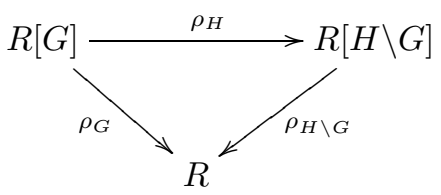

We will find an element $b \in R[G]$ such that $\rho_{H}\left(b^{2}\right)=-c^{2}$ for some $c \in R[H \backslash G], c \neq$ 0 . This will be a contradiction. For, on the one hand, $\rho_{G}\left(b^{2}\right) \geq 0$ by hypothesis $(\star)$. On the other hand, $\rho_{G}\left(b^{2}\right)=-\rho_{H \backslash G}\left(c^{2}\right)$ must be strictly negative by Lemma 4.4. 
We denote the dual action of $H$ on $G$ by

$$
\eta^{\prime}: R[G] \rightarrow R[H] \otimes R[G] .
$$

Let $X(H)$ be the character group of $H$ (an infinite cyclic group), and let $u \in X(H)$ be a non-trivial character. There exists an element $x \in R[G], x \neq 0$, with

$$
\eta^{\prime}(x)=u \otimes x .
$$

Indeed, if we decompose the $H$-module $R[G]$ into isotypical components,

$$
R[G]=\bigoplus_{\chi \in X(H)} R[G]_{(\chi)},
$$

then $R[G]_{(\chi)} \neq 0$ for every $\chi$, and it suffices to take any $0 \neq x \in R[G]_{(u)}$. Similarly, choose $0 \neq y \in R[G]$ with $\eta^{\prime}(y)=u^{-1} \otimes y$. Let $b:=x^{2}-y^{2} \in R[G]$. We have

$$
\eta^{\prime}\left(b^{2}\right)=u^{4} \otimes x^{4}-2 \cdot 1 \otimes\left(x^{2} y^{2}\right)+u^{-4} \otimes y^{4} .
$$

Since the Reynolds operator $R[H] \rightarrow R$ of $H$ sends $u^{n}$ to zero for all $n \neq 0$ (Example 4.3), we get for $\rho_{H}: R[G] \rightarrow R[H \backslash G]$, according to Lemma 2.11,

$$
\rho_{H}\left(b^{2}\right)=-2(x y)^{2}=-c^{2},
$$

where $c:=\sqrt{2} x y$ lies in $R[H \backslash G], c \neq 0$. By the argument given before, this completes the proof of Theorem 4.5 .

\section{Three EXAMPles}

To motivate our first example, we recall a result of Procesi and Schwarz for rational functions ([PS], Sect. 7, slightly generalized here):

Proposition 5.1. Let the reductive $R$-group $G$ act on the smooth irreducible affine $R$-variety $V$. Let $\pi: V \rightarrow V / / G=: W$ be the quotient morphism, and let $Z:=$ $\pi(V(R)) \subset W(R)$. Equivalent conditions are as follows:

(i) the set $Z$ is generically basic;

(ii) the preordering $T:=R(W) \cap \Sigma R(V)^{2}$ of the field $R(W)$ is finitely generated.

In fact, if $Z$ is generically equal to $\mathcal{S}_{W}\left(p_{1}, \ldots, p_{m}\right)$ with $p_{i} \in R[W]$, then $T$ is generated by $p_{1}, \ldots, p_{m}$ (as a preordering of $R(W)$ ). Moreover, conditions (i) and (ii) are satisfied when $G(R)$ is semi-algebraically compact.

Recall that $Z$ generically basic means that there exists a basic closed set $Z^{\prime}$ in $W(R)$ such that the set-theoretic difference of $Z$ and $Z^{\prime}$ is not Zariski dense in $W$.

Note that $R(W)$ may be smaller than $R(V)^{G}$, the field of $G$-invariant rational functions on $G$. Both coincide for all linear representation spaces $V$ of $G$ defined over $R$, if and only if every character $G \rightarrow \mathbb{G}_{m}$ defined over $R$ has finite image. In particular, $R(W)=R(V)^{G}$ is always true for linear representations $V$ if $G(R)$ is semi-algebraically compact. (These remarks were already made in [PS, 7.6.)

See [PS, 7.8 for a class of representations $V$, where the equivalent conditions of Proposition 5.1 fail.

5.2. Now consider the case where $G(R)$ is semi-algebraically compact. As we have just recalled, the preordering $\left(\Sigma R(V)^{2}\right)^{G}$ of $G$-invariant sums of squares of rational functions on $V$ is finitely generated in the field $R(V)^{G}=R(W)$. One is 
therefore wondering whether a similar result holds for regular functions. Thus, is the preordering

$$
S_{0}=\left(\Sigma R[V]^{2}\right)^{G}=\rho\left(\Sigma R[V]^{2}\right)
$$

in $R[V]^{G}=R[W]$ finitely generated? It turns out that this usually fails, as the following example shows.

Example 5.3. Consider the group $G$ of order two acting on $V=\mathbb{A}^{n}$ by $x \mapsto-x$. The ring of invariants $R[V]^{G}$ consists of all polynomials in $R[V]=R\left[x_{1}, \ldots, x_{n}\right]$ which contain only monomials of even degree. Thus, $R[V]^{G}$ is generated as an $R$ algebra by $R[V]_{2}$, the space of quadratic forms in $\left(x_{1}, \ldots, x_{n}\right)$. Identifying $R[V]_{2}$ with the space of symmetric $n \times n$-matrices over $R$, the cone $C:=S_{0} \cap R[V]_{2}$ consists of all psd symmetric matrices. If $S_{0}$ were finitely generated as a preordering in $R[V]^{G}$, then $C$ would be a polyhedral cone in $R[V]_{2}$, i.e. we would have $C=$ $R_{+} S_{1}+\cdots+R_{+} S_{m}$ with finitely many psd matrices $S_{\nu}$. But this is clearly not the case for $n \geq 2$.

On the other hand, if the same $G$ acts on $\mathbb{A}^{n}$ instead by

$$
\left(x_{1}, \ldots, x_{n}\right) \mapsto\left(x_{1}, \ldots, x_{n-1},-x_{n}\right),
$$

then $R[V]^{G}$ is generated by $u_{i}=x_{i}(i=1, \ldots, n-1)$ and $v=x_{n}^{2}$, and $S_{0}$ is the preordering generated by $v$.

5.4. Let $M \subset R[V]$ be a quadratic module, generated by $f_{1}, \ldots, f_{r}$, say. From the example in Remark 3.14 we know that $\rho(M)$ can be larger than the $S_{0}$-module generated by $\rho\left(f_{1}\right), \ldots, \rho\left(f_{r}\right)$. However, the question remains if $\rho(M)$ is at least finitely generated as an $S_{0}$-module. Our second example shows that the answer is usually negative, even if $M$ is a preordering and is $G$-invariant:

Example 5.5. Let $G=\mu_{2}$ act on $V=\mathbb{A}^{2}$ by interchanging the $x$ and $y$ coordinates, and let $T$ be the preordering in $R[V]=R[x, y]$ which is generated by $x$ and $y$. Then $T$ is $G$-invariant, but the $S_{0}$-module $\rho(T)$ fails to be finitely generated.

Assume to the contrary that $\rho(T)$ is finitely generated as an $S_{0}$-module. Then $\rho(T)$ is generated as an $S_{0}$-module by $1, x+y, x y$ and by finitely many polynomials of the form $h_{i}=2 \rho\left(x g_{i}^{2}\right)$ with non-constant $g_{i} \in R[x, y](i=1, \ldots, m)$. Consider the family of polynomials

$$
f_{r}=2 \rho\left((1-r y)^{2} x\right)=(x+y)-4 r x y+r^{2} x y(x+y),
$$

which lie in $\rho(T)$ for every value of the parameter $r \in R$. By assumption, for every $r \in R$ there exist $s_{0}, s_{1}, s_{2}, t_{1}, \ldots, t_{m} \in S_{0}$ with

$$
f_{r}=s_{0}+s_{1}(x+y)+s_{2} x y+\sum_{i=1}^{m} t_{i} h_{i} .
$$

A comparison of coefficients will lead to a contradiction. Let us discuss identity (2) for fixed $r$. Since $\operatorname{deg}\left(p_{1}+p_{2}\right)=\max \left\{\operatorname{deg}\left(p_{1}\right), \operatorname{deg}\left(p_{2}\right)\right\}$ for any two polynomials $p_{1}$, $p_{2} \in T$, each summand in (2) has degree $\leq 3$. In particular, $\operatorname{deg}\left(s_{0}\right) \leq 2, \operatorname{deg}\left(s_{2}\right) \leq$ 0 , and furthermore $\operatorname{deg}\left(g_{i}\right)=1$ and $t_{i} \in R_{+}$for each $i$. Writing $g_{i}=a_{i} x+b_{i} y+c_{i}$, the coefficient of $x^{3}$ on the right is $d+\sum_{i} t_{i} a_{i}^{2}$, where $d(\geq 0)$ is the coefficient of $x^{2}$ in $s_{1}$. Comparing with the left hand side we conclude $d=0$ (hence $s_{1} \in R_{+}$) and $t_{i} a_{i}=0$ for all $i$.

For any index $i$ with $t_{i} \neq 0$, we have

$$
h_{i}=b_{i}^{2} x y(x+y)+4 b_{i} c_{i} x y+c_{i}^{2}(x+y) .
$$


Comparing coefficients of $x^{2}$ on both sides of (2), we conclude that $s_{0}$ must be a scalar coefficient as well, and thus even $s_{0}=0$. Finally we compare coefficients of $x^{2} y, x y$ and $x$ in (2). This gives the identities

$$
\begin{aligned}
r^{2} & =\sum_{i} t_{i} b_{i}^{2}, \\
-4 r & =s_{2}+4 \sum_{i} t_{i} b_{i} c_{i}, \\
1 & =s_{1}+\sum_{i} t_{i} c_{i}^{2} .
\end{aligned}
$$

Writing $u=\left(b_{i} \sqrt{t_{i}}\right)_{i}$ and $v=\left(c_{i} \sqrt{t_{i}}\right)_{i}$ (two vectors in $\left.R^{m}\right)$ we conclude

$$
\|u\|^{2}=r^{2}, \quad\langle u, v\rangle \leq-r, \quad\|v\|^{2} \leq 1 .
$$

If $r>0$, the Cauchy-Schwarz inequality implies that both inequalities in (3) must be equalities. Therefore $u$ and $v$ must be linearly dependent, and hence $u= \pm r v$. There is an index $i$ with $t_{i} \neq 0$, and we conclude $r=\left|\frac{b_{i}}{c_{i}}\right|$.

But this shows that there are only finitely many values $r>0$ for which an identity (2) is possible with the fixed choice of generators. Hence we have proved that $\rho(T)$ is not finitely generated.

5.6. If $T$ is a $G$-invariant preordering in $R[V]$, then $\rho(T)$ is again a preordering, by Corollary 3.6. (c). Is this even true if we drop the assumption that $T$ is $G$-invariant? Our third example shows that the answer is no.

Example 5.7. Consider once more the group $G$ of order two, acting on $V=\mathbb{A}^{2}$ by $(x, y) \mapsto(y, x)$. Let $T$ be the preordering in $R[V]=R[x, y]$ generated by $g=1+x$ and $h=y^{2}+x$. We'll show that $\rho(g) \rho(h) \notin \rho(T)$, which implies that $\rho(T)$ is not a preordering. Suppose to the contrary that

$$
\rho(g) \rho(h)=\rho(t)
$$

for some $t \in T$. There exist sums of squares $p, q, r, s$ in $R[x, y]$ with $t=p+q g+$ $r h+s g h$. Again we have $\operatorname{deg}\left(f_{1}+f_{2}\right)=\max \left\{\operatorname{deg}\left(f_{1}\right), \operatorname{deg}\left(f_{2}\right)\right\}$ for any $f_{1}, f_{2}$ in the preordering generated by $T$ and $T^{\tau}$. Hence $\operatorname{deg}(p), \operatorname{deg}(q) \leq 2$ and $r, s \in R_{+}$. Evaluating both sides of (4) at the origin shows $p(0,0)=q(0,0)=0$, and so $p, q$ are homogeneous of degree two.

Consider the point $M:=(-1,0)$ and its conjugate $M^{\prime}=(0,-1)$ in the $(x, y)$ plane. To evaluate both sides of (44) at $M$, we record $g(M)=0, g\left(M^{\prime}\right)=1$, $h(M)=-1$ and $h\left(M^{\prime}\right)=1$. Hence $\rho(h)$ vanishes at $M$, and we get

$$
\begin{aligned}
0=\rho(t)(M) & =\rho(p)(M)+\rho(q g)(M)+\rho(r h)(M)+\rho(s g h)(M) \\
& =\frac{1}{2}\left(p(M)+p\left(M^{\prime}\right)\right)+\frac{1}{2} q\left(M^{\prime}\right)+\frac{1}{2} s .
\end{aligned}
$$

It follows that $p(M)=p\left(M^{\prime}\right)=q\left(M^{\prime}\right)=s=0$. Since $p$ is a psd quadratic form, we have $p=0$. Similarly, we get $q=a x^{2}$ with $a \geq 0$, which gives $2 \rho(q g)=$ $a\left(x^{2}+y^{2}+x^{3}+y^{3}\right)$. Re-writing (4) gives

$$
(2+x+y)\left(x^{2}+y^{2}+x+y\right)=2 a\left(x^{2}+y^{2}+x^{3}+y^{3}\right)+2 r\left(x+y+x^{2}+y^{2}\right),
$$

and comparing the coefficients of $x y$ we see a contradiction. 


\section{Moment PRoblems With SYMmetries}

In the second part of this paper we study moment problems on which a group of symmetries acts. Therefore, our ground field will now always be $R=\mathbb{R}$, the field of usual real numbers. Otherwise we'll keep the situation considered so far. So we have the reductive group $G$ over $\mathbb{R}$ which acts on the affine $\mathbb{R}$-variety $V$ via a morphism $G \times V \rightarrow V$ of varieties. The quotient morphism is $\pi: V \rightarrow V / / G=W$, where $W$ is the affine variety with $\mathbb{R}[W]=\mathbb{R}[V]^{G}$ (and $\pi$ is induced by the inclusion $\mathbb{R}[V]^{G} \subset \mathbb{R}[V]$ of rings). The image set of $\pi: V(\mathbb{R}) \rightarrow W(\mathbb{R})$ is denoted $Z$. By $\rho$ or $\rho_{V}$ we denote the Reynolds operator $\mathbb{R}[V] \rightarrow \mathbb{R}[W]$ (see Section 2.9).

6.1. Let $\mathbb{R}[V]^{\vee}$ denote the dual vector space of $\mathbb{R}[V]$, i.e. the space of all linear functionals $L: \mathbb{R}[V] \rightarrow \mathbb{R}$. The right action of $G(\mathbb{R})$ on $\mathbb{R}[V]$ induces a left action on $\mathbb{R}[V]^{\vee}$, namely $(g, L) \mapsto\left({ }^{g} L: f \mapsto L\left(f^{g}\right)\right)$. A linear functional $L \in \mathbb{R}[V]^{\vee}$ is called $G$-invariant if $L$ is invariant under this action of $G(\mathbb{R})$, i.e., if

$$
L\left(f^{g}\right)=L(f)
$$

holds for every $f \in \mathbb{R}[V]$ and $g \in G(\mathbb{R})$.

Lemma 6.2. A linear map $L \in \mathbb{R}[V]^{\vee}$ is $G$-invariant if and only if $L(f)=L(\rho f)$ for all $f \in \mathbb{R}[V]$. Hence the map

$$
\rho^{\vee}: \mathbb{R}[W]^{\vee} \rightarrow \mathbb{R}[V]^{\vee}, \quad F \mapsto F \circ \rho
$$

is an isomorphism from $\mathbb{R}[W]^{\vee}$ onto the space of $G$-invariant linear forms on $\mathbb{R}[V]$.

Proof. Consider the decomposition $\mathbb{R}[V]=\bigoplus_{\omega} \mathbb{R}[V]_{(\omega)}$ of the $G$-module $\mathbb{R}[V]$ into isotypical components, and let $\mathbb{R}[V]_{\left(\omega_{0}\right)}:=\mathbb{R}[V]^{G}=\mathbb{R}[W]$ denote the submodule of $G$-invariants. Any $G$-invariant linear form $L: \mathbb{R}[V] \rightarrow \mathbb{R}$ is a homomorphism of $G$ modules, where $\mathbb{R}$ is given the trivial $G$-module structure. But $\operatorname{Hom}_{G}(M, \mathbb{R})=\{0\}$ for any irreducible $G$-module $M \neq \mathbb{R}$. This shows that any $G$-invariant linear form $L$ vanishes on each $\mathbb{R}[V]_{(\omega)}, \omega \neq \omega_{0}$. Since $\rho$ is the projection of $\mathbb{R}[V]$ onto $\mathbb{R}[V]_{\left(\omega_{0}\right)}=\mathbb{R}[W]$, the lemma follows from this.

6.3. We are going to relate (Borel) measures on $W(\mathbb{R})$ to $G$-invariant (Borel) measures on $V(\mathbb{R})$. We only have results when $G(\mathbb{R})$ is compact. Given a measure $\mu$ on $V(\mathbb{R})$ for which every $f \in \mathbb{R}[V]$ is $\mu$-integrable, we write $L_{\mu}: f \mapsto \int_{V(\mathbb{R})} f d \mu$ for the linear functional "integration by $\mu$ ".

A measure $\mu$ on $V(\mathbb{R})$ will be called $G$-invariant if $\mu$ is invariant under translation by all elements of $G(\mathbb{R})$, i.e., if $\mu(g A)=\mu(A)$ for every Borel set $A \subset V(\mathbb{R})$ and every $g \in G(\mathbb{R})$. Note that this implies

$$
\int f d \mu=\int f^{g} d \mu
$$

for every $f \in \mathbb{R}[V]$ and $g \in G(\mathbb{R})$ (provided that either side exists). As usual, if $f: X \rightarrow Y$ is any continuous map of topological spaces and $\mu$ is a Borel measure on $X$, then $f_{*}(\mu)$ denotes the direct image measure on $Y$. It is characterized by $\left(f_{*} \mu\right)(B)=\mu\left(f^{-1}(B)\right)(B \subset Y$ any Borel set $)$.

We first recall a few basic relations between invariant measures on a $G$-space and measures on the orbit spaces. These facts must certainly be folklore among the experts. Since we have not been able to find suitable references, we decided to include the (easy) proofs. For the rest of this section, assume that the group $G(\mathbb{R})$ is compact. 
Lemma 6.4. Let $\sigma: G(\mathbb{R}) \times V(\mathbb{R}) \rightarrow V(\mathbb{R})$ be the group action. A Borel measure $\mu$ on $V(\mathbb{R})$ is $G$-invariant if and only if

$$
\mu=\sigma_{*}(\lambda \otimes \mu),
$$

where $\lambda$ is the normalized Haar measure on $G(\mathbb{R})$.

Proof. For $g \in G(\mathbb{R})$ let $l_{g}: x \mapsto g x$ denote left translation by $g$ on either $V(\mathbb{R})$ or $G(\mathbb{R})$. If $\mu=\sigma_{*}(\lambda \otimes \mu)$, then $\mu$ is $G$-invariant since

$$
\left(l_{g}\right)_{*}(\mu)=\left(l_{g} \circ \sigma\right)_{*}(\lambda \otimes \mu)=\left(\sigma \circ\left(l_{g} \times \mathrm{id}\right)\right)_{*}(\lambda \otimes \mu)=\sigma_{*}(\lambda \otimes \mu)=\mu .
$$

Conversely, if $\mu$ is $G$-invariant, then for each Borel set $B$ in $V(\mathbb{R})$ we have

$$
\begin{aligned}
\sigma_{*}(\lambda \otimes \mu)(B) & =(\lambda \otimes \mu)\left(\sigma^{-1}(B)\right) \\
& =\int_{G(\mathbb{R})} \mu\left(g^{-1} B\right) \lambda(d g)=\int_{G(\mathbb{R})} \mu(B) \lambda(d g)=\mu(B)
\end{aligned}
$$

by the Fubini formula.

Proposition 6.5. Let $\nu$ be any Borel measure on $Z=\operatorname{im}(\pi) \subset W(\mathbb{R})$.

(a) There exists a unique $G$-invariant Borel measure $\mu$ on $V(\mathbb{R})$ with $\pi_{*}(\mu)=$ $\nu$. We will denote it by $\mu=: \pi^{*}(\nu)$.

(b) Explicitly, if $f: V(\mathbb{R}) \rightarrow \mathbb{R}$ is a non-negative measurable function, then

$$
\int_{V(\mathbb{R})} f(x) \mu(d x)=\int_{Z} \bar{h}_{f}(y) \nu(d y)
$$

where the function $\bar{h}_{f}: Z \rightarrow \mathbb{R} \cup\{\infty\}$ is defined by

$$
\bar{h}_{f}(\pi x):=\int_{G(\mathbb{R})} f(g x) \lambda(d g)
$$

$(x \in V(\mathbb{R}))$.

Proof. Let $\lambda$ be the normalized Haar measure on $G(\mathbb{R})$. We will need the fact that $\lambda$ is invariant under left and right translation by elements of $G(\mathbb{R})$.

Given a Borel set $A$ in $V(\mathbb{R})$, let $x \in V(\mathbb{R})$, and write $o_{x}: G(\mathbb{R}) \rightarrow V(\mathbb{R})$ for the orbit map $o_{x}(g):=g x$. Put

$$
h_{A}(x):=\left(o_{x *} \lambda\right)(A)=\lambda\{g \in G(\mathbb{R}): g x \in A\} .
$$

The function $h_{A}: V(\mathbb{R}) \rightarrow[0,1]$ is measurable $\left(\left[\mathrm{Ba}\right.\right.$ 23.2). Since $o_{g x}=o_{x} \circ r_{g}$ (where $r_{g}: h \mapsto h g$ is right translation by $g \in G(\mathbb{R})$ ) we have

$$
h_{A}(g x)=\left(o_{x *} r_{g *} \lambda\right)(A)=\left(o_{x *} \lambda\right)(A)=h_{A}(x)
$$

for $x \in V(\mathbb{R})$ and $g \in G(\mathbb{R})$. Since the fibres of $\pi: V(\mathbb{R}) \rightarrow Z$ are precisely the $G(\mathbb{R})$-orbits (Theorem $2.5($ a) $), h_{A}$ induces a measurable function $\bar{h}_{A}: Z \rightarrow[0,1]$ by $\bar{h}_{A}(\pi(x))=h_{A}(x)(x \in V(\mathbb{R}))$.

Now let $\nu$ be a Borel measure on $Z$, and define

$$
\mu(A):=\int_{Z} \bar{h}_{A}(y) \nu(d y)
$$

for $A \subset V(\mathbb{R})$ a Borel set. Then $\mu$ is a Borel measure on $V(\mathbb{R})$, since if $A=\bigcup_{n \in \mathbb{N}} A_{n}$ is a countable union of pairwise disjoint Borel sets in $V(\mathbb{R})$, we have $h_{A}=\sum_{n} h_{A_{n}}$ 
(pointwise on $V(\mathbb{R})$ ), and therefore $\mu(A)=\sum_{n} \mu\left(A_{n}\right)$. Also $h_{g A}=h_{A}$ for $g \in G(\mathbb{R})$, and so $\mu$ is $G$-invariant. From the construction it is clear that $\pi_{*}(\mu)=\nu$.

Let $f: V(\mathbb{R}) \rightarrow \mathbb{R}_{+}$be measurable. The function $h_{f}: V(\mathbb{R}) \rightarrow \mathbb{R} \cup\{\infty\}, h_{f}(x):=$ $\int_{G(\mathbb{R})} f(g x) \lambda(d g)$ is again measurable and $G$-invariant, so it induces a measurable function $\bar{h}_{f}: Z \rightarrow \mathbb{R} \cup\{\infty\}$ as in the proposition. Given any $G$-invariant measure $\tilde{\mu}$ on $V(\mathbb{R})$ with $\pi_{*}(\tilde{\mu})=\nu$, we have

$$
\begin{aligned}
\int_{V(\mathbb{R})} f(x) \tilde{\mu}(d x) & =\int_{V(\mathbb{R})} \int_{G(\mathbb{R})} f(g x) \lambda(d g) \tilde{\mu}(d x) \\
& =\int_{V(\mathbb{R})} \bar{h}_{f}(\pi(x)) \mu(d x) \\
& =\int_{Z} \bar{h}_{f}(y) \pi_{*}(\mu)(d y) \\
& =\int_{Z} \bar{h}_{f}(y) \nu(d y)
\end{aligned}
$$

using $\tilde{\mu}=\sigma_{*}(\lambda \otimes \tilde{\mu})$ and Fubini's theorem. This establishes both the uniqueness of $\mu$ and the second part of the proposition.

Corollary 6.6. The operators $\pi_{*}$ and $\pi^{*}$ set up a bijective correspondence between the set of $G$-invariant Borel measures $\mu$ on $V(\mathbb{R})$ and the set of all Borel measures $\nu$ on $Z$. In particular, one has

$$
\pi^{*} \pi_{*}(\mu)=\mu \text { and } \pi_{*} \pi^{*}(\nu)=\nu .
$$

Corollary 6.7. Let $\nu$ be a Borel measure on $Z$, and let $\mu=\pi^{*} \nu$.

(a) For any $f \in \mathbb{R}[V]$ we have $\int_{V(\mathbb{R})} f d \mu=\int_{Z} \rho(f) d \nu$ (one integral exists iff the other exists).

(b) For any $G(\mathbb{R})$-invariant Borel set $A$ in $V(\mathbb{R})$ we have $\mu(A)=\nu(\pi(A)$ ).

(c) Given any $G$-invariant closed subset $K$ of $V(\mathbb{R})$, we have $\operatorname{supp}(\mu) \subset K$ iff $\operatorname{supp}(\nu) \subset \pi(K)$.

Proof. (b) is clear from $\nu=\pi_{*}(\mu)$ (again using the fact that the fibres of $\pi$ are the $G(\mathbb{R})$-orbits), and (c) follows from (b). As to (a), we have

$$
\int_{V(\mathbb{R})} f d \mu=\int_{Z} \bar{h}_{f}(y) d \nu(y)
$$

by Proposition 6.5] But $\bar{h}_{f}(\pi x)=\int_{G(\mathbb{R})} f(g x) d \lambda(g)$ is just $(\rho f)(x)$, for $x \in V(\mathbb{R})$; see Proposition 2.12, So $\bar{h}_{f}=\rho(f)$ as functions on $Z$.

Remark 6.8. In the situation of Corollary 6.7, assume that every function in $\mathbb{R}[V]$ is $\mu$-integrable (or equivalently, by Corollary 6.7 (a), that every function in $\mathbb{R}[W]$ is $\nu$-integrable). Then the linear forms $L_{\mu} \in \mathbb{R}[V]^{\vee}$ and $L_{\nu} \in \mathbb{R}[W]^{\vee}$ are related by

$$
L_{\mu}=L_{\nu} \circ \rho \text { and } L_{\nu}=\left.\left(L_{\mu}\right)\right|_{\mathbb{R}[W]} \text {. }
$$

Lemma 6.9. Let $K \subset V(\mathbb{R})$ be a $G$-invariant basic closed set, and let $L \in \mathbb{R}[V]^{\vee}$ be a $G$-invariant linear form. The following conditions are all equivalent:

(i) there is a measure $\mu$ on $K$ with $L(f)=\int_{K} f d \mu$ for every $f \in \mathbb{R}[V]^{G}$; 
(ii) there is a G-invariant measure $\mu$ on $K$ with $L(f)=\int_{K} f d \mu$ for every $f \in \mathbb{R}[V]$;

(iii) there is a measure $\nu$ on $\pi(K)$ with $L(f)=\int_{\pi(K)} f d \nu$ for every $f \in \mathbb{R}[W]$;

(iv) $L(f) \geq 0$ for every $f \in \mathcal{P}_{V}(K)^{G}=\mathcal{P}_{W}(\pi K)$.

Proof. (i) $\Rightarrow$ (iii): Assuming (i), let $\nu:=\pi_{*}(\mu)$. Then for every $f \in \mathbb{R}[W]$ we have $\int_{\pi(K)} f d \nu=\int_{K} f \circ \pi d \mu=L(f)$ by (i). (iii) $\Rightarrow$ (ii): Assuming (iii), put $\mu:=\pi^{*}(\nu)$. Then $\mu$ is $G$-invariant. Using Corollary 6.7(a) we have $\int_{K} f d \mu=\int_{\pi(K)} \rho(f) d \nu=$ $L(\rho(f))=L(f)$ for $f \in \mathbb{R}[V]$, where the last equality holds since $L$ is $G$-invariant (Lemma 6.2). The implication (ii) $\Rightarrow$ (i) is trivial.

By Haviland's theorem, (iii) is equivalent to $L(f) \geq 0$ for every $f \in \mathcal{P}_{W}(\pi K)$. By Corollary [3.9, $\mathcal{P}_{W}(\pi K)=\mathcal{P}_{V}(K)^{G}$.

Corollary 6.10. Let $L \in \mathbb{R}[V]^{\vee}$ be a linear functional on $\mathbb{R}[V]$ which is integration with respect to some Borel measure on $V(\mathbb{R})$. Then $L$ is $G$-invariant if and only if there exists a $G$-invariant Borel measure $\mu$ on $V(\mathbb{R})$ with $L=L_{\mu}$.

Proof. The 'if' part is obvious anyway. Conversely, if $\tilde{\mu}$ is some measure on $V(\mathbb{R})$ with $L=L_{\tilde{\mu}}$, then $\mu:=\pi^{*} \pi_{*} \tilde{\mu}$ is a $G$-invariant Borel measure with $L_{\mu}=L$ (see (i) $\Rightarrow$ (iii) $\Rightarrow$ (ii) in the last proof).

6.11. Given a basic closed set $K \subset V(\mathbb{R})$ and a quadratic module $M$ in $\mathbb{R}[V]$, recall that $M$ is said to solve the $K$-moment problem if the linear forms $L \in \mathbb{R}[V]^{\vee}$ with $\left.L\right|_{M} \geq 0$ are precisely the $K$-moment functionals, i.e., the linear forms represented by Borel measures on $K$. By Haviland's theorem, it is equivalent that the closure $\bar{M}$ of $M$ is equal to $\mathcal{P}_{V}(K)$. Here and in the sequel, the closure refers to the finest locally convex vector space topology on $\mathbb{R}[V]$. See [PSch], for example.

If, for given $K$, such $M$ can be found which is finitely generated (as a quadratic module), this allows a characterization of the $K$-moment functionals by an explicit recursive sequence of conditions. Indeed, if $M=\Sigma f_{1}+\cdots+\Sigma f_{r}$, say (with $\Sigma:=$ $\left.\Sigma \mathbb{R}[V]^{2}\right)$, then $L$ is a $K$-moment functional if and only if $L\left(q^{2} f_{i}\right) \geq 0$ for every $q \in \mathbb{R}[V]$ and $i=1, \ldots, r$; and for a fixed $i$, this translates into a positive semidefiniteness condition for a countable generalized Hankel matrix which depends in a direct explicit way on $L$ and $f_{i}$.

Following $[\mathrm{Sm}$, we say that $M$ has the strong moment property (SMP) if the closure $\bar{M}$ of $M$ is saturated. Thus $M$ solves the $K$-moment problem iff $M$ has $(\mathrm{SMP})$ and $X_{V}(M)=\widetilde{K}$.

We are now going to study variants of this notion which take the group action into account. The idea is that, while it may be hard or even impossible to characterize all moment functionals of Borel measures on $K$, the task may become easier if one only aims at characterizing the invariant moment functionals.

Definition 6.12. Let $K$ be a $G$-invariant basic closed set in $V(\mathbb{R})$, and let $N$ be a quadratic module in $\mathbb{R}[W]=\mathbb{R}[V]^{G}$. We'll say that $N$ solves the invariant $K$-moment problem if the following is true for every $G$-invariant linear functional $L: \mathbb{R}[V] \rightarrow \mathbb{R}:$

Conditions (i)-(iv) of Lemma 6.9 hold for $L$ if and only if $L(f) \geq 0$ for every $f \in N$. 
Corollary 6.13. Let $K \subset V(\mathbb{R})$ be basic closed and $G$-invariant, and let $N$ be a quadratic module in $\mathbb{R}[W]$. The following are equivalent:

(i) $N$ solves the invariant $K$-moment problem;

(ii) $\bar{N}=\mathcal{P}_{W}(\pi K)$;

(iii) $N$ solves the (usual) $\pi(K)$-moment problem in $W$.

Proof. The equivalence of (ii) and (iii) is well-known (and recalled in Section6.11). The equivalence of (i) and (iii) is clear from Lemma 6.9

6.14. We also introduce a weakening of this notion. Again let $K$ be a $G$-invariant basic closed set in $V(\mathbb{R})$, and let $M$ be a quadratic module in $\mathbb{R}[V]$. We say that $M$ solves the averaged $K$-moment problem if the following is true for every $G$-invariant linear functional $L: \mathbb{R}[V] \rightarrow \mathbb{R}:$

Conditions (i)-(iv) of Lemma 6.9 hold for $L$ if and only if $L(f) \geq 0$ for every $f \in M$.

Corollary 6.15. Let $K \subset V(\mathbb{R})$ be basic closed and $G$-invariant, and let $M$ be a quadratic module in $\mathbb{R}[V]$. The following are equivalent:

(i) $M$ solves the averaged $K$-moment problem;

(ii) $\overline{\rho(M)}=\mathcal{P}_{W}(\pi K)$;

(iii) $\rho(M)$ solves the (usual) $\pi(K)$-moment problem in $W$.

Proof. (i) $\Leftrightarrow$ (ii) is clear from Lemma 6.9, Again, (ii) $\Leftrightarrow$ (iii) is well-known (see Section 6.11).

Remark 6.16. Let $K \subset V(\mathbb{R})$ be $G$-invariant, and let $N$ be a quadratic module in $\mathbb{R}[W]$. If $N$ solves the invariant $K$-moment problem, then $K$ is determined by $N$ via $K=\mathcal{S}_{V}(N)$. Similarly, if $M \subset \mathbb{R}[V]$ solves the averaged $K$-moment problem, then $K=\mathcal{S}_{V}(M)$.

Keeping this in mind, Corollary 6.13 justifies the following terminology: A quadratic module $N$ in $\mathbb{R}[W]$ has the invariant moment property (IMP) if $\bar{N}$ is saturated in $\mathbb{R}[W]$, that is, if $N$ has the (SMP) (in $\mathbb{R}[W]$ ).

Similarly, Corollary 6.15 justifies us to say: A quadratic module $M$ in $\mathbb{R}[V]$ has the averaged moment property $(A M P)$ if $\overline{\rho(M)}$ is saturated in $\mathbb{R}[W]$, that is, if $\rho(M)$ has the (SMP) (in $\mathbb{R}[W]$ ).

Thus, $M$ has the (AMP) if and only if $\rho(M)$ has the (IMP). Of course, we are usually only interested in (IMP) or (AMP) when the corresponding module is finitely generated.

Proposition 6.17. Let $K \subset V(\mathbb{R})$ be a $G$-invariant basic closed set, and let $M$ be a quadratic module in $\mathbb{R}[V]$ which solves the (usual) $K$-moment problem in $\mathbb{R}[V]$. Then $M$ has the averaged moment property (AMP).

Proof. The hypothesis says $\bar{M}=\mathcal{P}_{V}(K)$. Application of the Reynolds operator yields

$$
\rho(M) \subset \rho\left(\mathcal{P}_{V}(K)\right)=\rho(\bar{M}) \subset \overline{\rho(M)} .
$$

Since $\rho\left(\mathcal{P}_{V}(K)\right)=\mathcal{P}_{W}(\pi K)$ (Corollary $[3.9)$ is closed, this implies $\overline{\rho(M)}=\mathcal{P}_{W}(\pi K)$. 
Remark 6.18. We now compare the various moment properties considered. Let $K$ be a $G$-invariant basic closed set in $V(\mathbb{R})$, let $N$ be a quadratic module in $\mathbb{R}[V]^{G}=\mathbb{R}[W]$, and let $M$ be the quadratic module in $\mathbb{R}[V]$ generated by $N$. We consider the following three properties of $N$ :

$\left(\operatorname{IMP}_{K}\right) N$ solves the invariant $K$-moment problem;

$\left(\mathrm{AMP}_{K}\right) M$ solves the averaged $K$-moment problem;

$\left(\mathrm{SMP}_{K}\right) M$ solves the (ordinary) $K$-moment problem (in $V$ ).

Corollary 6.19. We have the implications $\left(S M P_{K}\right) \Rightarrow\left(A M P_{K}\right)$ and $\left(I M P_{K}\right) \Rightarrow$ $\left(A M P_{K}\right)$. On the other hand, neither $\left(I M P_{K}\right)$ nor $\left(A M P_{K}\right)$ implies $\left(S M P_{K}\right)$, in general.

Proof. The first implication follows by Proposition 6.17. The second implication holds since $\bar{N}=\mathcal{P}_{W}(\pi K)$ and $N \subset \rho(M) \subset \mathcal{P}_{W}(\pi K)$ (Proposition 3.12) imply $\overline{\rho(M)}=\mathcal{P}_{W}(\pi K)$. Examples 7.1 and 7.2 show that $\left(\operatorname{IMP}_{K}\right)$ does not imply $\left(\mathrm{SMP}_{K}\right)$. Therefore, $\left(\mathrm{AMP}_{K}\right)$ does not imply $\left(\mathrm{SMP}_{K}\right)$ either.

We were not able to decide whether $\left(\mathrm{SMP}_{K}\right)$ implies $\left(\operatorname{IMP}_{K}\right)$, nor whether $\left(\mathrm{AMP}_{K}\right)$ implies $\left(\mathrm{IMP}_{K}\right)$. See open question 1.

Remark 6.20. In a similar vein, one may consider the following three properties of the $G$-invariant basic closed set $K$ :

(IMP) The invariant $K$-moment problem is finitely solvable;

(AMP) the average $K$-moment problem is finitely solvable;

(SMP) the (ordinary) $K$-moment problem is finitely solvable.

Of course, "finitely solvable" means solvable by finitely generated quadratic modules, in $\mathbb{R}[V]$ for (AMP) and (SMP), and in $\mathbb{R}[W]$ for (IMP).

Clearly, (SMP) $\Rightarrow($ AMP) and (IMP) $\Rightarrow($ AMP). Sections 7.1 and 7.2 show (IMP) $\nRightarrow(\mathrm{SMP})$ (and therefore (AMP) $\nRightarrow(\mathrm{SMP})$ ). Section 7.3 (due to Tim Netzer) shows that $(\mathrm{SMP}) \nRightarrow(\mathrm{IMP})$ (and therefore (AMP) $\nRightarrow(\mathrm{IMP})$ ).

Remark 6.21. For describing the $G$-invariant linear functionals $L \in \mathbb{R}[V]^{\vee}$ which correspond to measures on $K$ (or equivalently, to $G$-invariant such measures; see Lemma 6.9), (IMP) and (AMP) are in principle as good as (SMP). Indeed, (AMP) and (SMP) both mean that one has to check $L\left(q^{2} f_{i}\right) \geq 0$ for all $q \in \mathbb{R}[V]$ and a finite number of fixed $f_{i} \in \mathbb{R}[V]$. For (IMP) it is the same, except that the $f_{i}$ are in addition $G$-invariant and one may restrict to $G$-invariant multipliers $q$, which further simplifies the task. Therefore, from a practical point of view, it is interesting to find situations where (IMP) or (AMP) holds, but (SMP) fails.

Such situations are constructed in Examples 7.1 and 7.2 Here (SMP) fails, while (IMP) (and thus (AMP)) holds.

Remark 6.22. If $K$ is a $G$-invariant basic closed set in $V(\mathbb{R})$, then the $K$-moment problem need not be solvable by any number of $G$-invariant functions.

In other words, what we are claiming is that the preordering $T$ in $\mathbb{R}[V]$ generated by $\mathcal{P}_{V}(K)^{G}=\mathcal{P}_{W}(\pi K)$ need not be dense in $\mathcal{P}_{V}(K)$, although the saturation of $T$ is clearly equal to $\mathcal{P}_{V}(K)$.

Indeed, in Examples 7.1 or 7.2 there exists a finitely generated preordering $N$ in $\mathbb{R}[W]$ satisfying $\bar{N}=\mathcal{P}_{W}(\pi K)$. Let $M$ be the preordering generated by $N$ in $\mathbb{R}[V]$. Then $\mathcal{P}_{W}(\pi K)=\bar{N} \subset \bar{M}$, and therefore also $\bar{T} \subset \bar{M}$. On the other hand, 
$\bar{M} \subsetneq \mathcal{P}_{V}(K)$, since $M$ is finitely generated, but the $K$-moment problem is not finitely solvable in these examples.

Other classes of examples illustrating the point of this remark will be constructed in CKM].

Our last result is of a negative character. It provides a large class of cases where the ordinary $K$-moment problem is not finitely solvable, and where the invariant $K$-moment problem is not finitely solvable either:

Theorem 6.23. Let the finite group $G$ act on the irreducible normal affine $\mathbb{R}$ variety $V$, and let $K$ be a $G$-invariant basic closed set in $V(\mathbb{R})$. Suppose that there exists a $G$-equivariant completion $V \hookrightarrow X$ of $V$ such that

(1) $X$ is normal and projective,

(2) for every irreducible component $X^{\prime}$ of $X-V, \bar{K} \cap X^{\prime}(\mathbb{R})$ is Zariski dense in $X^{\prime}$.

Let $W=V / / G$. Then every finitely generated quadratic module $N$ in $\mathbb{R}[W]$ with $\mathcal{S}_{W}(N)=\pi(K)$ is stable and closed. In particular, if $\operatorname{dim}(V) \geq 2$, then the $G$ invariant $K$-moment problem is not finitely solvable.

Proof. Note that in (2), $\bar{K}$ denotes the closure of $K$ inside $X(\mathbb{R})$. The variety $X$ has a covering by open affine $G$-invariant subsets, since every $G$-orbit is contained in some open affine set $U \subset X$, and since the subset $\bigcap_{g \in G} g U$ is affine and $G$-invariant. Therefore one can form the (geometric) quotient variety $Y=X / G$. The morphism $\pi: X \rightarrow Y$ is finite, and $Y$ is a normal and complete variety $([\mathrm{Kr}]$, p. 100). The inclusion $V \hookrightarrow X$ induces an open embedding $W \hookrightarrow Y$. The square of morphisms

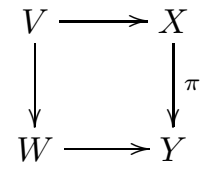

is cartesian.

Write $X_{0}:=X-V$ and $Y_{0}:=Y-W$. Every irreducible component $Y^{\prime}$ of $Y_{0}$ has the form $Y^{\prime}=\pi\left(X^{\prime}\right)$ with some irreducible component $X^{\prime}$ of $X_{0}$. We claim that $Y^{\prime}(\mathbb{R}) \cap \overline{\pi(K)}$ (the closure taken in $Y(\mathbb{R})$ ) is Zariski dense in $Y^{\prime}$.

Indeed, by hypothesis (2) there exist $\beta \in \widetilde{K}$ and $\alpha \in \widetilde{X^{\prime}(\mathbb{R})}$ with support of $\alpha$ equal to $X^{\prime}$ and with $\beta \succ \alpha$ (a specialization in $\widetilde{X(\mathbb{R})}$, the real spectrum of $X$ ). Applying the map $\tilde{\pi}: \widetilde{X(\mathbb{R})} \rightarrow \widetilde{Y(\mathbb{R})}$ of real spectra we get $\widetilde{\pi}(\beta) \succ \widetilde{\pi}(\alpha)$. Moreover, $\tilde{\pi}(\beta) \in \widetilde{\pi(K)}$, and the support of $\widetilde{\pi}(\alpha)$ is $\pi\left(X^{\prime}\right)=Y^{\prime}$, which proves the claim.

The affine variety $W=V / / G$ is again normal. So we can apply [PSch], Theorem 2.14 to $W$ and its completion $Y$, and to the basic closed subset $\pi(K)$ of $W(\mathbb{R})$. By this result, every quadratic module $N$ in $\mathbb{R}[W]$ with $\mathcal{S}_{W}(N)=\pi(K)$ is stable and closed. Since $\operatorname{dim}(K)=\operatorname{dim}(V)$ (as follows from (2)), $N$ cannot have the (SMP) if $\operatorname{dim}(V) \geq 2$, by [Sch3], Thm. 5.4. By Corollary 6.13, this means that $N$ cannot solve the invariant $K$-moment problem.

Example 6.24. The proposition applies in particular when $V$ is a linear representation space of $G$ and the set $K$ contains a non-empty open cone. Indeed, every linear $G$-action on $V=\mathbb{A}^{n}$ extends to a linear $G$-action on $X=\mathbb{P}^{n}$. 


\section{MORE EXAMPLES}

Let $K$ be a basic closed semi-algebraic set in $V(\mathbb{R})$ which is $G$-invariant. We consider the finite solvability of the $K$-moment problem on the one hand, and of the $G$-invariant $K$-moment problem on the other. In general, the question of characterizing the solutions of the moment problem will become easier when one restricts his or her attention to solutions with symmetries. Our first examples are meant to demonstrate this fact. They show that finite characterizations of the $G$ invariant solutions may be available at the same time when such characterizations do not exist for the class of all solutions.

Here is a first class of examples in dimension one.

7.1. Let $f(x)$ be a square-free monic polynomial in $\mathbb{R}[x]$ of degree $d$, and consider the set $K=\left\{(x, y) \in \mathbb{R}^{2}: y^{2}=f(x)\right\}$. The group $G$ of order two acts on $K$ by $(x, y) \mapsto(x,-y)$. The usual $K$-moment problem fails to be finitely solvable if $d \geq 3$, whereas the $G$-invariant $K$-moment problem can be finitely solved.

Indeed, $K$ is the set of $\mathbb{R}$-points of an affine non-singular hyperelliptic curve $C$, whose genus $g=\left\lfloor\frac{d-1}{2}\right\rfloor$ is positive for $d \geq 3$. All points of $C$ at infinity are real (there are one or two of them, according to whether $d$ is odd or even). Hence the $K$-moment problem is not finitely solvable (see [PSch], Corollary 3.10).

On the other side, the quotient curve $C / / G$ is the affine line, and $\pi: C \rightarrow$ $C / / G=\mathbb{A}^{1}$ is the map $\pi(x, y)=x$. Hence $Z=\{x \in \mathbb{R}: f(x) \geq 0\}$, and the $Z$-moment problem is solved by a finite number of polynomials in $x$, which are explicit in terms of the real zeros of $f(x)$ (see [Sch1, 5.23.1 or [KM], 2.2). So these polynomials solve the $G$-invariant $K$-moment problem.

Many variations of this example can be built, using proper $G$-invariant closed subsets $K$ of $C(\mathbb{R})$.

7.2. For a two-dimensional example consider the dihedral group $G=D_{4}$ of order eight acting on the real affine plane $V=\mathbb{A}^{2}$ in the natural way (as the symmetry group of a square centered at the origin). The basic closed set

$$
K:=\left\{(x, y) \in \mathbb{R}^{2}:-1 \leq\left(x^{2}-1\right)\left(y^{2}-1\right) \leq 0\right\}
$$

in the plane $\mathbb{R}^{2}=V(\mathbb{R})$ is $G$-invariant.

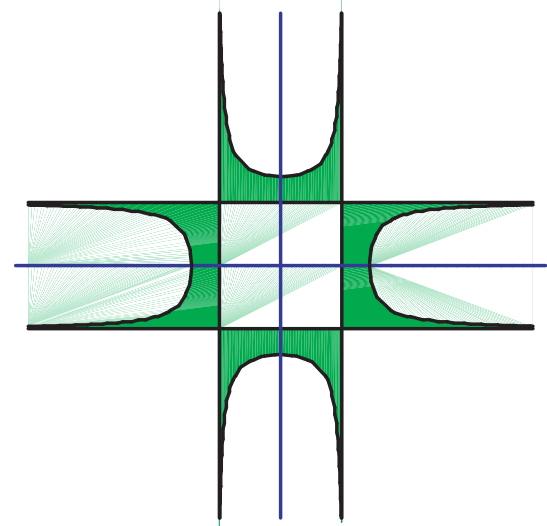

The ring of invariants is $\mathbb{R}[x, y]^{G}=\mathbb{R}[u, v]$ with

$$
u=x^{2}+y^{2}, \quad v=x^{2} y^{2},
$$


and $W=\mathbb{A}^{2} / / G$ is itself an affine plane (see $[\underline{\mathrm{St}}]$ ). The image of $\pi: V(\mathbb{R}) \rightarrow W(\mathbb{R})$ is

$$
Z=\pi\left(\mathbb{R}^{2}\right)=\left\{(u, v) \in \mathbb{R}^{2}: u \geq 0, v \geq 0, u^{2} \geq 4 v\right\} .
$$

Since $\left(x^{2}-1\right)\left(y^{2}-1\right)=v-u+1$, we have

$$
\pi(K)=\left\{(u, v) \in \mathbb{R}^{2}: v \geq 0,1 \leq u-v \leq 2\right\} .
$$

This is a (half-) strip in the $(u, v)$-plane:

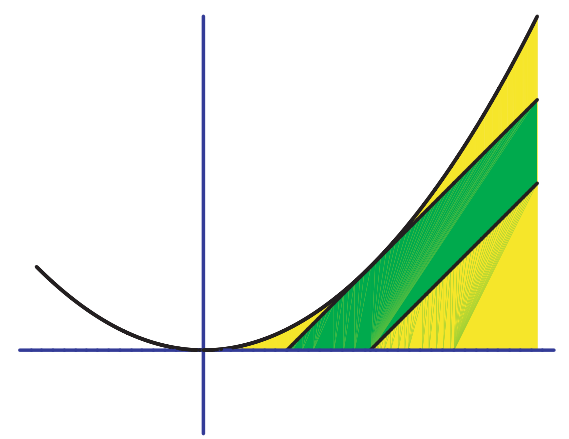

The moment problem for $\pi(K)$ is solved by the preordering $N$ in $\mathbb{R}[u, v]=W(\mathbb{R})$ generated by $v, u-v-1$ and $2-u+v$ (see KMS, Corollary 5.2). This means that the $G$-invariant $K$-moment problem is solved by $N$ (Corollary 6.13 ).

On the other hand, we'll now show that the usual $K$-moment problem is not finitely solvable. Given a real parameter $c$, consider the affine plane curve

$$
E_{c}:\left(x^{2}-1\right)\left(y^{2}-1\right)=c .
$$

Let $\mathbb{P}^{2}=\mathbb{A}^{2} \cup L$ be the projective plane, where $L$ is the line at infinity. An easy calculation shows that the Zariski closure $\bar{E}_{c}$ of $E_{c}$ in $\mathbb{P}^{2}$ meets $L$ in two points $P$ and $Q$, both real, and furthermore $P$ and $Q$ are ordinary double points of $\bar{E}_{c}$ (with real tangents).

Moreover, the affine curve $E_{c}$ is non-singular for $c \neq 0,1$. For these values, therefore, $E_{c}$ is a non-singular affine curve of genus one which has four points at infinity, all of them real. Since $E_{c}(\mathbb{R}) \subset K$ for $-1 \leq c<0$, we conclude that the $K$-moment problem is not finitely solvable, using [PSch, Corollary 3.10. (One single such value $c$ is already enough for the argument.)

We are grateful to Tim Netzer for finding the following example and allowing us to include it here.

7.3. The following two-dimensional example shows that (SMP) does not imply (IMP). In particular, (AMP) does not imply (IMP).

Let the group $G$ of order two act on the affine plane $V=\mathbb{A}^{2}$ by permuting the coordinates $x$ and $y$. The basic closed set

$$
K:=\left\{(x, y) \in \mathbb{R}^{2}: x \geq 0, y \geq 0, x y \leq 1\right\}
$$

is $G$-invariant. Furthermore, the preordering generated by $x, y, 1-x y$ in $\mathbb{R}(V)=$ $\mathbb{R}[x, y]$ solves (SMP) for $K$; see for example [KMS, Example 8.4. 
The ring of $G$-invariant polynomials $\mathbb{R}[x, y]^{G}$ is a polynomial ring $\mathbb{R}[u, v]$, where $u=x+y$ and $v=x y$. So $W:=\mathbb{A}^{2} / / G$ is again an affine plane, and the image of $\pi: V(\mathbb{R}) \rightarrow W(\mathbb{R})$ is

$$
Z=\pi\left(\mathbb{R}^{2}\right)=\left\{(u, v) \in \mathbb{R}^{2}: u^{2} \geq 4 v\right\}
$$

One checks that

$$
\pi(K)=\left\{(u, v) \in \mathbb{R}^{2}: 0 \leq u, 0 \leq v \leq 1, u^{2} \geq 4 v\right\}
$$

holds.

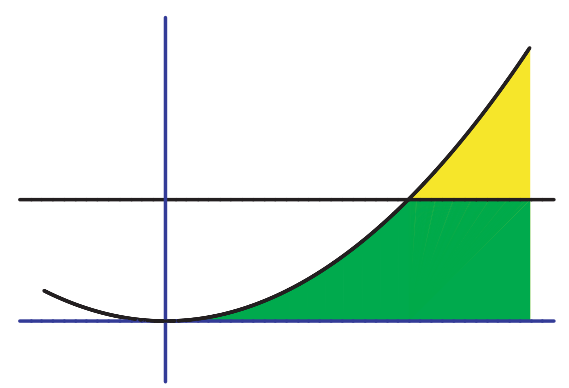

Lemma 7.4. The $\pi(K)$-moment problem is not finitely solvable.

Proof. Suppose there are polynomials $f_{1}, \ldots, f_{s} \in \mathbb{R}[u, v]$ such that the preordering $P O\left(f_{1}, \ldots, f_{s}\right)$ solves (SMP) for $\pi(K)$. Then for any $b \in[0,1]$, the preordering

$$
P O\left(f_{1}(u, b), \ldots, f_{s}(u, b)\right) \subset \mathbb{R}[u]
$$

solves (SMP) for the set

$$
[2 \sqrt{b}, \infty[\subset \mathbb{R}
$$

by [Sch3], Prop. 4.8. By [KM], Theorems 2.1 and 2.2, the natural generator for this set, namely $u-2 \sqrt{b}$, must be among the $f_{i}(u, b)$ up to a constant factor. So without loss of generality, assume

$$
f_{1}(u, b)=r(b)(u-2 \sqrt{b})
$$

for infinitely many $b \in[0,1]$ and some positive function $r$. Writing

$$
f_{1}(u, v)=\sum_{j} g_{j}(v) u^{j}
$$

and comparing coefficients, we get $g_{0}(b)=-2 r(b) \sqrt{b}$ and $g_{1}(b)=r(b)$ for infinitely many $b \in[0,1]$. So for all these $b$,

$$
g_{0}(b)^{2}=4 r(b)^{2} b=4 g_{1}(b)^{2} b,
$$

so $g_{0}^{2}=4 g_{1}^{2} v$ in $\mathbb{R}[v]$. As the left hand side has even degree and the right hand side has odd degree, this is a contradition.

Remark 7.5. Note that the example does not give a negative answer to the question of whether $\left(\mathrm{SMP}_{K}\right)$ implies $\left(\mathrm{IMP}_{K}\right)$. For this one would need to have a collection of finitely many $G$-invariant polynomials which solve the $K$-moment problem. It can be shown that such a collection does not exist.

So the question of whether $\left(\mathrm{SMP}_{K}\right)$ implies $\left(\mathrm{IMP}_{K}\right)$ remains open. 


\section{OPEN QUESTIONS}

1. Let $K \subset V(\mathbb{R})$ be basic closed and $G$-invariant. Assume that $N$ is a quadratic module in $\mathbb{R}[V]^{G}=\mathbb{R}[W]$ and that $M$ is the quadratic module generated by $N$ in $\mathbb{R}[V]$. If $M$ solves the $K$-moment problem (on $V$ ), does it follow that $N$ solves the $\pi(K)$-moment problem (on $W)$ ? In other words, does the implication $\left(\mathrm{SMP}_{K}\right) \Rightarrow$ $\left(\mathrm{IMP}_{K}\right)$ hold (cf. Remark 6.18 and Corollary 6.19)? In all cases where $\left(\mathrm{SMP}_{K}\right)$ is satisfied and that we could analyze, it turned out that $\left(\operatorname{IMP}_{K}\right)$ holds as well. Similarly, we do not know any example where $\left(\mathrm{AMP}_{K}\right)$ holds and $\left(\mathrm{IMP}_{K}\right)$ fails.

2. What about the averaged moment property (AMP) in the situations covered by Theorem 6.23, in particular Example 6.24. Can it possibly hold for $\operatorname{dim}(V) \geq 2$ ?

3. Given $G$ with $G(R)$ semi-algebraically compact, consider linear representation spaces $V$ of $G$ (or more general affine $G$-varieties). Under what conditions on $V$ is $S_{0}=\left(\Sigma R[V]^{2}\right)^{G}$ finitely generated as a preordering in $R[V]^{G}$ ? See Example 5.3 .

4. Assume that $K \subset V(R)$ is $G$-invariant and that the saturated preordering $\mathcal{P}_{V}(K)$ is finitely generated. Does this imply that $\mathcal{P}_{V}(K)^{G}=\mathcal{P}_{W}(\pi K)$ is finitely generated as well (as a preordering in $R[W]=R[V]^{G}$ )?

5. How can Example 7.2 be generalized, and what is its essential feature? Note that for such an example in $V=\mathbb{A}^{n}, n \geq 2$, the failure of the moment property for $K$ in $V$ cannot be due to the containment of a cone in $K$, by the negative result of Example 6.24.

6. (Cf. Remark 6.22) Let $K$ be a $G$-invariant basic closed set in $V(\mathbb{R})$, and let $T$ be the preordering generated in $\mathbb{R}[V]$ by $\mathcal{P}_{V}(K)^{G}=\mathcal{P}_{W}(\pi K)$. Then

$$
T \subset \mathcal{P}_{V}(K)=\operatorname{Sat}_{V}(T)
$$

Note that $T=\mathcal{P}_{V}(K)$ means that every $f \in R[V]$ with $\left.f\right|_{K} \geq 0$ can be written $f=\sum_{i} a_{i}^{2} f_{i}$ with $a_{i}, f_{i} \in R[V],\left.f_{i}\right|_{K} \geq 0$ and $f_{i} G$-invariant. This motivates the following: Find necessary and sufficient conditions so that $T=\mathcal{P}_{V}(K)$, or so that $T$ is dense in $\mathcal{P}_{V}(K)$. Note that, in general, $T$ fails to be dense in $\mathcal{P}_{V}(K)$ (Remark 6.22).

\section{REFERENCES}

[Ba] H. Bauer: Maß- und Integrationstheorie. 2. Aufl. De Gruyter, Berlin, 1992. MR1181881 (93g:28001)

[Bi] D. Birkes: Orbits of linear algebraic groups. Ann. Math. (2) 93, 459-475 (1971). MR0296077 (45:5138)

[BCR] J. Bochnak, M. Coste, M.-F. Roy: Real Algebraic Geometry. Erg. Math. Grenzgeb. (3) 36, Springer, Berlin, 1998. MR1659509 (2000a:14067)

[Bo] A. Borel: Linear Algebraic Groups. Second enlarged edition. Grad. Texts Math. 126, Springer, New York, 1991. MR1102012 (92d:20001)

[Br] L. Bröcker: On symmetric semialgebraic sets and orbit spaces. In: Singularities Symposium — Łojasiewicz 70 (Kraków, 1996). Banach Center Publ. 44, Polish Acad. Sci., Warszawa, 1998, pp. 37-50. MR1677398 (2000b:14076)

[CKM] J. Cimprič, S. Kuhlmann, M. Marshall: Positivity in power series rings (2008), to appear in: Advances in Geometry.

[DK] H. Derksen, G. Kemper: Computational Invariant Theory. Encycl. Math. Sciences, Springer, Berlin, 2002. MR1918599 (2003g:13004)

[Ga] F. R. Gantmacher: Matrix Theory. Chelsea Publishing, New York, 1960. MR0107649 $(21: 6372 \mathrm{c})$

[GP] K. Gatermann, P. A. Parrilo: Symmetry groups, semidefinite programs, and sums of squares. J. Pure Appl. Algebra 192, 95-128 (2004). MR2067190(2005d:68155) 
[HP] J. W. Helton, M. Putinar: Positive polynomials in scalar and matrix variables, the spectral theorem and optimization. In: Vol. Operator Theory, Structured matrices and dilations, Theta, Bucharest, June 2007, pp. 229-306. MR2389626

[KS] M. Knebusch, C. Scheiderer: Einführung in die reelle Algebra. Vieweg, Braunschweig/ Wiesbaden, 1989. MR 1029278 (90m:12005)

[KH] B. Kostant, G. Hochschild: Differential forms and Lie algebra cohomology for algebraic linear groups. Illinois J. Math. 6, 264-281 (1962). MR0139695 (25:3126)

[Kr] H. Kraft: Geometrische Methoden in der Invariantentheorie. Aspects of Mathematics, Vieweg, Braunschweig/Wiesbaden, 1984. MR768181 (86j:14006)

[KM] S. Kuhlmann, M. Marshall: Positivity, sums of squares and the multi-dimensional moment problem. Trans. Am. Math. Soc. 354, 4285-4301 (2002). MR1926876 (2003j:14078)

[KMS] S. Kuhlmann, M. Marshall, N. Schwartz: Positivity, sums of squares and the multi-dimensional moment problem II. Adv. Geom. 5, 583-607 (2005). MR 2174483 (2006i:14064)

[Lu] D. Luna: Sur certains opérations différentiables des groupes de Lie. Am. J. Math. 97, 172-181 (1975). MR0364272 (51:527)

[PSch] V. Powers, C. Scheiderer: The moment problem for non-compact semialgebraic sets. Adv. Geom. 1, 71-88 (2001). MR.1823953(2002c:14086)

[PW] V. Powers, Th. Wörmann: An algorithm for sums of squares of real polynomials. J. Pure Appl. Algebra 127, 99-104 (1998). MR1609496 (99a:11047)

[PD] A. Prestel, Ch. N. Delzell: Positive Polynomials. Monographs in Mathematics, Springer, Berlin, 2001. MR,1829790 (2002k:13044)

[PS] C. Procesi, G. Schwarz: Inequalities defining orbit spaces. Invent. Math. 81, 539-554 (1985). MR807071 (87h:20078)

[Sch1] C. Scheiderer: Sums of squares on real algebraic curves. Math. Z. 245, 725-760 (2003). MR.2020709 (2004k:14103)

[Sch2] C. Scheiderer: Positivity and sums of squares: A guide to some recent results. Preprint 2003, available at www.ihp-raag.org/publications.

[Sch3] C. Scheiderer: Non-existence of degree bounds for weighted sums of squares representations. J. Complexity 21, 823-844 (2005). MR2182447|(2006k:14117)

[Sch4] C. Scheiderer: Sums of squares on real algebraic surfaces. Manuscr. Math. 119, 395-410 (2006). MR2223624 (2006m:14079)

[Sch5] C. Scheiderer: Local study of two-dimensional preorderings. In progress.

[Sm] K. Schmüdgen: On the moment problem of closed semi-algebraic sets. J. Reine Angew. Math. 558, 225-234 (2003). MR1979186 (2004e:47019)

[St] B. Sturmfels: Algorithms in Invariant Theory. Springer-Verlag, Vienna, 1993. MR.1255980 (94m:13004)

Faculty of Mathematics and Physics, University of Ljubljana, Jadranska 19, SI-1000 LuUbluana, Slovenija

E-mail address: cimpric@fmf.uni-lj.si

URL: http://www.fmf.uni-lj.si/ cimpric

Department of Mathematics and Statistics, University of Saskatchewan, Room 142 McLean Hall, 106 Wiggins Road, Saskatoon, Saskatchewan, Canada S7N 5E6

E-mail address: skuhlman@snoopy.usask.ca

$U R L:$ http://math.usask.ca/〜skuhlman

Fachbereich Mathematik und Statistik, Universität Konstanz, D-78457 Konstanz, GERmany

E-mail address: claus.scheiderer@uni-konstanz.de

URL: http://www.math.uni-konstanz.de/ scheider 